\title{
Analysis of Heterosis and Combining Ability for Yield and Yield Contributing traits in Medium Maturity Hybrids of Maize (Zea mays L.)
}

\author{
Deshraj Gurjar $^{1 *}$ and Rambabu Dubey ${ }^{2}$ \\ ${ }^{1}$ Lovely Professional University Phagwara, Punjab, India \\ ${ }^{2}$ Rajasthan college of Agriculture, Mpuat, Udaipur, India \\ *Corresponding author
}

\section{Keywords}

Maize, Line X

Tester Analysis, Per

Se Performance,

Combining Ability

and Heterosis

Article Info

Accepted:

12 November 2020

Available Online:

10 December 2020

\section{A B S T R A C T}

The present investigation was under taken with a view to estimate the extent of heterosis, per se performance, combining ability. The hybrids were attempted by adopting line $\mathrm{x}$ tester mating design among 15 inbred lines and 3 testers in maize (Zea mays L.) during Rabi 2015. These were evaluated during Kharif 2016 and Rabi 2016-17 in three environments in randomized block design with three replications. Data were recorded for fifteen traits. Analysis of variance revealed that mean squares due to genotypes $\mathrm{x}$ environments and its components viz., parents $\mathrm{x}$ environments, crosses $\mathrm{x}$ environments and parents $\mathrm{v} / \mathrm{s}$ crosses $\mathrm{x}$ environments were significant for majority of the characters. This indicated the presence of considerable amount of variability in the present set of genotypes. An overall per se performance indicated that parental line $\mathrm{L}_{10}$ exhibited maximum mean for grain yield per plant $(88.20 \mathrm{~g})$ along with maximum mean for harvest index $(39.32 \%)$ and minimum mean value for days to 75 per cent brown husk (104.56 days). The hybrid $L_{2} \times T_{1}$ exhibited maximum mean value for grain yield per plant (163.13 $\mathrm{g} /$ plant) and exhibited maximum estimate of significant economic heterosis in $\mathrm{E}_{1}$ $(36.12 \%), \mathrm{E}_{2}(12.47 \%), \mathrm{E}_{3}(34.58 \%)$ and on pooled basis $(28.29 \%)$ indicating dominance of genes for higher grain yield per plant. This hybrid also showed significant estimate of economic heterosis for grain protein content in $\mathrm{E}_{1}(6.40 \%), \mathrm{E}_{2}(3.95 \%)$ and over the environment $(4.41 \%)$. Out of 45 hybrids five best hybrids which exhibited positive significant sca effects for grain yield per plant are viz., $\mathrm{L}_{2} \times \mathrm{T}_{1}, \mathrm{~L}_{7} \times \mathrm{T}_{2}, \mathrm{~L}_{10} \times \mathrm{T}_{3}, \mathrm{~L}_{1} \times \mathrm{T}_{3}$ and $L_{9} \times T_{1}$. Hybrid $L_{2} \times T_{1}$ also exhibited higher magnitude of economic heterosis with higher mean performance. These hybrids $\mathrm{L}_{2} \times \mathrm{T}_{1}, \mathrm{~L}_{7} \times \mathrm{T}_{2}, \mathrm{~L}_{10} \times \mathrm{T} 3, \mathrm{~L}_{1} \times \mathrm{T}_{3}$, and $\mathrm{L}_{9} \times \mathrm{T}_{1}$ were crosses between poor $\mathrm{x}$ good gca effects of parent for grain yield per plant.

\section{Introduction}

In India, maize is the third important food crop after rice and wheat. According to latest data (2015-16), it is being cultivated on 8.69 $\mathrm{m}$ ha with $80 \%$ area during kharif season. The current maize production is $21.7 \mathrm{mt}$, with an average productivity of $2.5 \mathrm{t} / \mathrm{ha}$. Despite maize being predominantly rainfed crop its productivity is more than rice which is mainly grown under assured irrigated/rainfed conditions. Maize contributes nearly $9 \%$ in the national food basket and more than 400 billion to the agricultural GDP at current 
prices. In addition it generates employment to over 1000 million man days at the farm and downstream agricultural and industrial sectors. Maize is primarily used for feed $(60 \%)$ followed by human food (24\%), industrial (starch) products (14\%) and bevarages and seed (1\% each). Thus, maize has attained an important position as industrial crop because $75 \%$ of its produce is used in starch and feed industries. In India, maize is predominantly cultivated as rainfed crop but due to focused research on single cross hybrids (SCH) since 2006, the productivity of maize has increased @ 134 $\mathrm{kg} / \mathrm{ha} / \mathrm{annum}$. The adoption of $\mathrm{SCH}$ technology in India is around $60 \%$, with this adoption of technology it has registered a growth rate of more than $7 \%$ in production and $6 \%$ in productivity in last five years. Study of combining ability is important for selecting parents for hybridization. Sprague and Tatum (1942), first time proposed the concepts of general combining ability (gca) and specific combining ability (sca). According to them, GCA variance is due to additive variance and SCA variance is due to non-additive variance, both act as an important diagnostic tool in selection of suitable parents and cross combination. Among the various design used for combining ability analysis, Line $\mathrm{x}$ Tester analysis (Kempthorne, 1957) has been extensively used to assess the combining ability of parents and crosses for different quantitative characters as well as to study the extent of heterosis for yield, yield contributing characters in maize.

\section{Materials and Methods}

The experimental was conducted in three locations viz., Instructional Farm during Kharif 2016 and Rabi 2016-17, Rajasthan College of Agriculture, Udaipur (Rajasthan) and Agriculture Research Sub Station, Vallabhnagar, Udaipur and the pooled analysis result data are given below the materials consisted of 45 hybrids obtained by crossing of 15 lines viz., EI-2303, EI -2308, EI-2315, EI-2317, EI-2325, EI-2414, EI2431, EI-2440, EI-2446, EI-2508, EI-2516, EI-2517, EI-2546, EI-2547 and 3 testers EI586-3-1, EI-BML-6, EI-670-2. The hybrids and parents were evaluated in randomized block design with three replications in four meter row with spacing of $60 \times 25 \mathrm{~cm}$. All the recommended agronomical practices and plant protection measures were followed as and when required to raise a good crop of maize. Five plants in parents and hybrids were randomly selected in each replication and observations were recorded for days to $50 \%$ tasseling, days to $50 \%$ silking, AnthesisSiliking interval, 75 per cent brown husk, plant height $(\mathrm{cm})$, ear height $(\mathrm{cm})$, cob length (cm), number of grain rows per cob, cob girth (cm), 100 grain weight (gm), harvest index $(\%)$, grain yield per plant (gm), Grain Protein content $(\%)$, Grain starch content $(\%)$ and Grain oil content (\%). The mean values were subjected to line $\mathrm{x}$ tester analysis as suggested by Kempthorne (1957).

\section{Results and Discussion}

The analysis of variance for experimental design (Table 2 to 3 ) revealed that the presence of significant amount of variability for parents in individual environment as well as pooled over the environments for all the characters except days to 50 per cent silking in $E_{3}$, days to 75 per cent brown husk in $E_{2}$ and $E_{3}$, plant height in $E_{3}$, cob length in $E_{2}$, cob girth in $E_{3}, 100$-grain weight in $E_{1}$. This suggested that the parental lines selected were quite variable for most of characters under study. The mean squares due crosses were significant in individual environment and over the environments for all the characters. This results suggested that the presence of considerable amount of variability among the hybrids. 
Mean squares due parents' v/s crosses were significant for all the characters in all three environments as well as pooled over the environment except days to 50 per cent silking in $\mathrm{E}_{3}$, anthesis silking interval in $\mathrm{E}_{1}$, days to 75 per cent brown husk in $\mathrm{E}_{1} \& \mathrm{E}_{3}$, plant height were non-significant. This indicated the existence of applicable amount of a genetic variability in the experimental material of present.

Under pooled analysis of variance mean squares due to genotypes $\mathrm{x}$ environments, were significant for all the characters except days to 50 per cent tasseling, days to 50 per cent silking, days to 75 per cent brown husk, ear height were non-significant, mean squares due to parents $x$ environments were significant for all the characters except days to 50 per cent tasseling, days to 50 per cent silking, days to 75 per cent brown husk, plant height, ear height, cob length, cob girth, 100grain weight, harvest index, mean squares due to crosses $\mathrm{x}$ environments were significant for all the characters except days to 50 per cent tasseling, days to 50 per cent silking, days to 75 per cent brown husk, ear height, number of grain rows per ear were non-significant and mean squares due to parents $\mathrm{v} / \mathrm{s}$ crosses $\mathrm{x}$ environments were significant for all the characters except days to 50 per cent tasseling, days to 50 per cent silking, days to 75 per cent brown husk, plant height, ear height, cob girth, 100-grain weight were nonsignificant.

Similar trends for variances and its components in maize were reported by Dubey et al., (2001), Joshi et al., (2002), Firoz et al., (2007), Lal et al., (2007), Dubey et al., (2009), Sharief et al., (2009), Sumalini and Shobha Rani (2010), Premlatha et al.(2011), Yousif and Sedeeq (2011), Abuali et al., (2012), Lal and Kumar (2012), Anusheela et al.(2013), Abrha et al., (2013), Singh et al.(2013), Motamedi et al.(2014), Rajesh et al., (2014), Rastgari et al., (2014) and Ruswandi (2015) for grain yield, quality traits and brown husk traits.

Combining ability referred as ability of a parent to transmit its performance to its offsprings. As combining ability often depend upon complex interaction systems among genes, certain combination nick well produce superior off-springs, whereas other involving equally promising parent produce disappointing progeny.

Sprague and Tatum (1942) produced the concept of general and specific combining ability as measure of gene action through their investigation in corn. They defined the term general combining ability (gca) as average performance of a line set of hybrids, which can be recognized as measure of additive gene action including additive $\mathrm{x}$ additive interactions.

The specific combining ability (sca) refers those instances when certain cross combinations do relatively better or worse than would be expected on the basis of average performance of parental lines involved. This is controlled by non-additive genetic variance including dominance and epistatic interactions.

With advancement of biometrical genetics, several method have been developed to assess the general and specific combining ability of different genetic material, viz., top cross technique which was proposed by Jenkins and Brunson (1932) or Davis (1927), polly cross by Tysdal et al., (1942), diallel cross by Griffing (1956) and Hayman (1954), line x tester mating design by Kempthorne (1957), partial diallel cross by Kempthorne and Curnow (1961), triallel cross by Rawlings and Cocherham (1962) and modified line x tester by Murty et al., (1967). 
Table.1 Pooled analysis of variance for different characters in maize

\begin{tabular}{|c|c|c|c|c|c|c|c|c|c|c|}
\hline SN & Source & df & $\begin{array}{l}\text { Days to } 50 \% \\
\text { tasseling }\end{array}$ & $\begin{array}{l}\text { Days to } 50 \% \\
\text { silking }\end{array}$ & $\begin{array}{c}\text { Antheis - } \\
\text { silking } \\
\text { interval }\end{array}$ & $\begin{array}{c}\text { Days to } 75 \% \\
\text { brown husk }\end{array}$ & $\begin{array}{l}\text { Plant height } \\
\text { (cm) }\end{array}$ & $\begin{array}{c}\text { Ear height } \\
\quad(\mathrm{cm})\end{array}$ & $\begin{array}{l}\text { Cob Length } \\
\text { (cm) }\end{array}$ & $\begin{array}{c}\text { Number of } \\
\text { Grain rows/ear }\end{array}$ \\
\hline 1. & Environment & 2 & $238682.27 * *$ & $262597.05^{* *}$ & $572.84 * *$ & $360045.40^{* *}$ & $10826.19 * *$ & 57.03 & $258.70^{* *}$ & $106.90 * *$ \\
\hline \multirow[t]{10}{*}{3.} & Genotype & 66 & $93.28 * *$ & $88.78 * *$ & $2.08 * *$ & $66.22 * *$ & $1051.11 * *++$ & $937.70 * *$ & $11.81 * *++$ & $21.32 * *++$ \\
\hline & Check & 3 & $58.69 * *$ & $51.44 * *$ & $0.92 * *$ & $1.44 * *$ & $137.68 * *$ & $961.30 * *$ & $5.22 * *$ & $2.58 * *$ \\
\hline & P vs Chk & 1 & $0.34 * *$ & $1.98 * *$ & $0.67 *$ & $2.22 * *$ & $1869.53 * *$ & $4541.81 * *$ & $36.67 * *$ & $191.28 * *$ \\
\hline & Tester & 2 & $23.59 * *$ & $16.33 * *$ & $4.15 * *$ & $27.11 * *$ & $229.85 * *$ & $100.04 * *$ & $3.08 * *$ & $3.52 * *$ \\
\hline & Line & 14 & $65.17 * *$ & $50.92 * *$ & $3.92 * *$ & $27.01 * *$ & $564.71 * *$ & $418.38 * *$ & $7.45 * *$ & $6.18 * *$ \\
\hline & $\mathrm{T} \mathrm{v/s} \mathrm{L}$ & 1 & $4.59 * *$ & $3.21 * *$ & 0.12 & $4.30 * *$ & $47.78 * *$ & $1704.48 * *$ & $0.00 * *$ & $27.83 * *$ \\
\hline & $\mathrm{P}$ v/s C & 1 & $117.15^{* *}$ & $91.17 * *$ & $1.63 * *$ & $58.72 * *$ & $27.32 * *$ & $1328.93 * *$ & $88.90 * *$ & $748.48 * *+$ \\
\hline & Cross & 44 & $111.05 * *$ & $110.46 * *$ & $1.54 * *$ & $87.94 * *$ & $1322.26 * *++$ & $1057.51 * *$ & $12.65^{* *++}$ & $11.62 * *$ \\
\hline & Tester & 2 & $663.12 * *$ & $665.56 * *$ & $1.22 * *$ & $433.01 * *$ & $6219.03 * *$ & $10606.27 * *$ & $18.42 * *$ & $18.20 * *+$ \\
\hline & Line & 14 & $122.98 * *$ & $109.55 * *$ & $1.55 * *$ & $88.39 * *$ & $709.22 * *$ & $590.10 * *$ & $13.73^{* *++}$ & $14.02 * *$ \\
\hline \multirow{11}{*}{4.} & Check x E & 6 & 0.53 & 2.69 & $2.92 * *$ & 4.55 & 100.90 & 3.32 & $2.34 *$ & 0.43 \\
\hline & Chk x P x E & 2 & 1.55 & 3.34 & $0.49 *$ & 8.17 & 221.47 & 28.68 & 2.65 & 0.86 \\
\hline & $P \times E$ & 34 & 0.87 & 2.49 & $2.54 * *$ & 6.81 & 151.38 & 13.51 & 1.09 & $2.62 * *$ \\
\hline & $\mathrm{T} \times \mathrm{E}$ & 4 & 1.48 & 5.44 & $3.98 * *$ & 6.56 & 23.90 & 22.81 & 0.95 & 0.57 \\
\hline & $L \times E$ & 28 & 0.75 & 2.23 & $2.43 * *$ & 6.90 & 176.24 & 12.17 & 1.17 & $3.09 * *$ \\
\hline & $\mathrm{T}$ v/s L x E & 2 & 1.30 & 0.23 & $1.15^{* *}$ & 6.06 & 58.26 & 13.58 & 0.22 & 0.10 \\
\hline & $\mathrm{P}$ v/s C x E & 2 & 5.07 & 5.58 & $3.98 * *$ & 1.52 & 145.48 & 35.99 & $9.37 * *$ & $3.31 *$ \\
\hline & Cross x E & 88 & 3.73 & 4.74 & $1.90 * *$ & 7.40 & $363.66 * *$ & 12.64 & $3.20 * *$ & 0.96 \\
\hline & $\mathrm{T} \times \mathrm{E}$ & 4 & 7.33 & 9.64 & $4.07 * *$ & 5.58 & 22.45 & 25.87 & $5.62 * *$ & $2.18^{*}$ \\
\hline & $L \times E$ & 28 & 3.86 & 3.71 & $1.18 * *$ & 8.46 & $407.46 * *$ & 9.04 & $3.30 * *$ & 0.52 \\
\hline & $\mathrm{L} \times \mathrm{T} \times \mathrm{E}$ & 56 & 3.41 & 4.90 & $2.10 * *$ & 7.00 & $366.14 * *$ & 13.49 & $2.97 * *$ & 1.09 \\
\hline 5. & Pooled Error & 396 & 5.51 & 5.59 & 0.11 & 10.65 & 156.03 & 27.07 & 1.01 & 0.88 \\
\hline 5. & Bartlet & 2 & $69.85 * *$ & $70.34 * *$ & $118.09 * *$ & $49.17 * *$ & 3.46 & $9.20 *$ & $19.99 * *$ & $10.78 * *$ \\
\hline
\end{tabular}


Table.1 Continued...

\begin{tabular}{|c|c|c|c|c|c|c|c|c|c|}
\hline SN & Source & df & Cob girth (cm) & $\begin{array}{c}\text { 100-grain } \\
\text { weight }(\mathrm{g}) \text { : }\end{array}$ & $\begin{array}{c}\text { Harvest index } \\
(\%)\end{array}$ & $\begin{array}{l}\text { Grain Yield / } \\
\text { Plant (g) }\end{array}$ & $\begin{array}{l}\text { Grain protein } \\
\text { content in }(\%)\end{array}$ & $\begin{array}{c}\text { Grain starch } \\
\text { content in }(\%)\end{array}$ & $\begin{array}{c}\text { Grain Oil } \\
\text { content in }(\%)\end{array}$ \\
\hline 1. & Environment & 2 & $4.94 * *$ & $101.53 * *$ & $206.40 * *$ & $21883.04 * *$ & $5.91 * *$ & $49.18 * *$ & $5.31 * *$ \\
\hline 2. & Rep./Env & 6 & $3.33 * *$ & $62.25 * *$ & $21.80 * *$ & $155.38 *$ & $0.14 * *$ & $1.60 * *$ & $0.06 * *$ \\
\hline \multirow[t]{11}{*}{3.} & Genotype & 66 & $1.95 * *++$ & $586.52 * *++$ & $142.35 * *++$ & $8718.03 * *++$ & $3.85^{* *++}$ & $68.97 * *++$ & $1.22 * *++$ \\
\hline & Check & 3 & $0.02 * *$ & $3.61 * *$ & $5.44 * *$ & $199.27 * *$ & $0.64 * *+$ & $35.58 * *++$ & $0.55 * *++$ \\
\hline & P vs Chk & 1 & $10.28 * *$ & $8260.37 * *$ & $1329.45 * *$ & $91059.46 * *+$ & $18.56 * *+$ & $3.27 * *$ & $0.82 * *$ \\
\hline & Parent & 17 & $0.37 * *$ & $31.04 * *$ & $89.80 * *$ & $869.90 * *++$ & $4.36 * *++$ & $115.91 * *++$ & $1.53 * *++$ \\
\hline & Line & 14 & $0.41 * *+$ & $33.62 * *$ & $72.34 * *$ & $775.98 * *++$ & $5.07 * *++$ & $116.04 * *++$ & $1.47 * *++$ \\
\hline & $\mathrm{T} \mathrm{v} / \mathrm{s} \mathrm{L}$ & 1 & $0.37 * *$ & $37.36 * *$ & $512.62 * *$ & $1539.92 * *$ & $0.15^{* *}$ & $245.34 * *+$ & $0.95 * *$ \\
\hline & $\mathrm{P}$ v/s C & 1 & $48.63 * *$ & $31191.39 * *$ & $6622.65 * *+$ & $306628.71 * *+$ & $81.65^{* *+}+$ & $129.51 * *+$ & $0.57 * *$ \\
\hline & Cross & 44 & $1.66 * *++$ & $139.26 * *++$ & $26.61 * *$ & $5485.67 * *++$ & $2.17 * *++$ & $53.16 * *++$ & $1.18 * *++$ \\
\hline & Tester & 2 & $3.12 * *$ & $626.07 * *$ & $47.48 * *$ & $10738.79 * *$ & $0.22 * *$ & $2.21 * *$ & $0.74 * *$ \\
\hline & Line & 14 & $2.18 * *$ & $187.51 * *++$ & $24.67 * *$ & $7104.46 * *++$ & $3.53 * *++$ & $45.37 * *++$ & $1.69 * *++$ \\
\hline & $\mathrm{L} \times \mathrm{T}$ & 28 & $1.30 * *++$ & $80.36^{* *++}$ & $26.09 * *$ & $4301.06 * *++$ & $1.63 * *++$ & $60.70 * *++$ & $0.96 * *++$ \\
\hline \multirow{11}{*}{4.} & Check x E & 6 & 0.13 & 1.50 & 1.72 & 86.82 & $0.10 * *$ & $1.04 * *$ & $0.03 * *$ \\
\hline & Chk x P x E & 2 & 0.14 & 4.00 & 13.52 & $627.64 * *$ & $0.45 * *$ & $1.30 * *$ & 0.01 \\
\hline & $\mathrm{P} \times \mathrm{E}$ & 34 & 0.14 & 4.68 & 2.69 & $117.64 * *$ & $0.08 * *$ & $2.57 * *$ & $0.04 * *$ \\
\hline & $T \times E$ & 4 & 0.01 & 0.44 & 3.39 & 53.19 & 0.01 & $10.41 * *$ & $0.03 * *$ \\
\hline & $L \times E$ & 28 & $0.16 *$ & 4.89 & 2.44 & $113.08 * *$ & $0.09 * *$ & $0.86 * *$ & $0.03 * *$ \\
\hline & $\mathrm{T}$ v/s L x E & 2 & 0.15 & 10.25 & 4.89 & $310.41 * *$ & $0.04 * *$ & $10.75 * *$ & $0.25 * *$ \\
\hline & $\mathrm{P}$ v/s C x E & 2 & 0.15 & 3.48 & $67.53 * *$ & $975.12 * *$ & $2.24 * *$ & $0.92 *$ & $0.59 * *$ \\
\hline & Cross x E & 88 & $0.14 *$ & $9.02 * *$ & $25.87 * *$ & $336.51 * *$ & $0.09 * *$ & $1.03 * *$ & $0.03 * *$ \\
\hline & $\mathrm{T} \times \mathrm{E}$ & 4 & 0.23 & 9.70 & $43.28 * *$ & $1699.76 * *$ & $0.08 * *$ & $0.61 *$ & $0.12 * *$ \\
\hline & $L \times E$ & 28 & 0.09 & $9.09 *$ & $25.09 * *$ & $273.02 * *$ & $0.09 * *$ & $1.15^{* *}$ & $0.02 * *$ \\
\hline & L x T x E & 56 & $0.15^{*}$ & $8.94 * *$ & $25.02 * *$ & $270.88 * *$ & $0.09 * *$ & $1.01 * *$ & $0.02 * *$ \\
\hline 5. & Pooled Error & 396 & 0.10 & 5.40 & 6.94 & 61.06 & 0.01 & 0.21 & 0.00 \\
\hline 5. & Bartlet & 2 & $7.89 *$ & 2.15 & $10.76^{* *}$ & 3.70 & 1.88 & $25.00 * *$ & $12.58 * *$ \\
\hline
\end{tabular}


Table.2 GCA and SCA effects for days to 50 per cent tasseling and days to per cent silking

\begin{tabular}{|c|c|c|c|c|c|c|c|c|c|}
\hline \multirow[t]{2}{*}{ SN } & \multirow[t]{2}{*}{ Genotype } & \multicolumn{4}{|c|}{ Days to 50 per cent tasseling } & \multicolumn{4}{|c|}{ Days to 50 per cent silking } \\
\hline & & E1 & E2 & E3 & Pool & E1 & E2 & E3 & Pool \\
\hline 1 & $\mathrm{~T}_{1}$ & $-1.62 * *$ & $-1.80^{* *}$ & $-2.44 * *$ & $-1.96 * *$ & $-1.72 * *$ & $-1.73^{* *}$ & $-2.21 * *$ & $-1.89 * *$ \\
\hline 2 & $\mathrm{~T}_{2}$ & -0.47 & -0.53 & -0.36 & -0.45 & -0.36 & -0.47 & -0.85 & $-0.56^{*}$ \\
\hline 3 & $\mathrm{~T}_{3}$ & $2.09 * *$ & $2.33 * *$ & $2.80 * *$ & $2.41 * *$ & $2.08 * *$ & $2.20 * *$ & $3.06 * *$ & $2.45^{* *}$ \\
\hline 4 & $\mathrm{~L}_{1}$ & -0.13 & -0.78 & 1.47 & 0.19 & -0.45 & -0.67 & 1.06 & -0.02 \\
\hline 5 & $\mathrm{~L}_{2}$ & -0.24 & 0.00 & -0.31 & -0.19 & -0.23 & -0.11 & -0.39 & -0.24 \\
\hline 6 & $\mathrm{~L}_{3}$ & $1.53^{*}$ & $2.00^{* * *}$ & $2.69^{*}$ & $2.07 * *$ & 0.99 & $2.00^{* * *}$ & $3.06^{* *}$ & $2.02 * *$ \\
\hline 7 & $\mathrm{~L}_{4}$ & $-2.58 * *$ & $-3.33^{* *}$ & -2.09 & $-2.67 * *$ & $-2.34 * *$ & $-3.11 * *$ & -1.94 & $-2.46^{* *}$ \\
\hline 8 & $\mathrm{~L}_{5}$ & $-2.58 * *$ & $-2.44 * *$ & $-4.87 * *$ & $-3.30 * *$ & $-2.56^{* *}$ & $-2.33^{* *}$ & $-4.61 * *$ & $-3.17 * *$ \\
\hline 9 & $\mathrm{~L}_{6}$ & 0.87 & $1.44^{*}$ & 1.02 & $1.11^{*}$ & 0.99 & 1.00 & 0.50 & 0.83 \\
\hline 10 & $\mathrm{~L}_{7}$ & 0.64 & 0.89 & 0.69 & 0.74 & 0.88 & 0.78 & 0.39 & 0.68 \\
\hline 11 & $\mathrm{~L}_{8}$ & -0.13 & -0.67 & -0.53 & -0.44 & -0.12 & -0.22 & 0.61 & 0.09 \\
\hline 12 & $\mathrm{~L}_{9}$ & $1.42 *$ & $1.44^{*}$ & $2.80^{*}$ & $1.89 * *$ & $1.21^{*}$ & $1.89 * *$ & 1.73 & $1.61 * *$ \\
\hline 13 & $\mathrm{~L}_{10}$ & $-1.80 * *$ & $-2.44 * *$ & $-2.53 *$ & $-2.26 * *$ & $-1.67 * *$ & $-2.22 * *$ & $-2.39 *$ & $-2.09 * *$ \\
\hline 14 & $\mathrm{~L}_{11}$ & 0.42 & 1.22 & 1.36 & $1.00^{*}$ & 0.55 & 0.89 & 1.06 & 0.83 \\
\hline 15 & $\mathrm{~L}_{12}$ & $-3.69 * *$ & $-4.00^{* * *}$ & $-4.64 * *$ & $-4.11 * *$ & $-3.23 * *$ & $-3.89^{* *}$ & $-4.72 * *$ & $-3.95^{* *}$ \\
\hline 16 & $\mathrm{~L}_{13}$ & $2.76^{* *}$ & $3.22 * *$ & 1.91 & $2.63^{* *}$ & $2.55^{* *}$ & $2.78^{* *}$ & 1.50 & $2.28 * *$ \\
\hline 17 & $\mathrm{~L}_{14}$ & $1.98 * *$ & $2.44 * *$ & 1.91 & $2.11 * *$ & $2.10^{* * *}$ & $2.33^{* *}$ & $2.50^{*}$ & $2.31^{* *}$ \\
\hline 18 & $\mathrm{~L}_{15}$ & $1.53^{*}$ & 1.00 & 1.13 & $1.22 * *$ & $1.33^{*}$ & 0.89 & 1.61 & $1.28 * *$ \\
\hline 19 & $\mathrm{~L}_{1} \times \mathrm{T}_{1}$ & 1.62 & -1.09 & -2.11 & -0.53 & 2.16 & -1.04 & -3.68 & -0.85 \\
\hline 20 & $\mathrm{~L}_{2} \times \mathrm{T}_{1}$ & 0.40 & 0.80 & 2.00 & 1.07 & -0.06 & 1.07 & 3.10 & 1.37 \\
\hline 21 & $\mathrm{~L}_{3} \times \mathrm{T}_{1}$ & $2.62 *$ & $2.80^{*}$ & 3.33 & $2.92 * *$ & $2.72 *$ & 2.29 & 2.65 & $2.55^{* *}$ \\
\hline 22 & $\mathrm{~L}_{4} \times \mathrm{T}_{1}$ & 1.07 & $2.47 *$ & 1.4 & 1.66 & 0.72 & 2.40 & 0.99 & 1.37 \\
\hline 23 & $\mathrm{~L}_{5} \times \mathrm{T}_{1}$ & 1.40 & 0.91 & -0.78 & 0.51 & 1.61 & 1.62 & 0.99 & 1.40 \\
\hline 24 & $\mathrm{~L}_{6} \times \mathrm{T}_{1}$ & -0.71 & -0.64 & -1.00 & -0.79 & -1.28 & -0.04 & -0.46 & -0.60 \\
\hline 25 & $\mathrm{~L}_{7} \times \mathrm{T}_{1}$ & 0.51 & 0.91 & 3.00 & 1.47 & 0.16 & 1.18 & 3.32 & 1.55 \\
\hline 26 & $\mathrm{~L}_{8} \times \mathrm{T}_{1}$ & 0.62 & -0.20 & -0.44 & -0.01 & 0.83 & -0.49 & 0.10 & 0.15 \\
\hline 27 & $\mathrm{~L}_{9} \times \mathrm{T}_{1}$ & $-3.27 * *$ & $-2.64 *$ & -2.44 & $-2.79 * *$ & $-3.50 * *$ & $-3.27 * *$ & -3.35 & $-3.37 * *$ \\
\hline 28 & $\mathrm{~L}_{10} \times \mathrm{T}_{1}$ & 0.96 & 2.24 & 4.22 & $2.47 * *$ & 1.39 & 2.18 & 3.76 & $2.44 * *$ \\
\hline 29 & $\mathrm{~L}_{11} \times \mathrm{T}_{1}$ & -0.27 & -0.42 & -0.67 & -0.45 & -0.84 & 0.40 & -0.01 & -0.15 \\
\hline 30 & $\mathrm{~L}_{12} \times \mathrm{T}_{1}$ & 1.18 & 2.13 & 1.33 & 1.55 & 1.27 & 1.84 & 1.10 & 1.40 \\
\hline 31 & $\mathrm{~L}_{13} \times \mathrm{T}_{1}$ & $-3.27 * *$ & $-3.09 *$ & -2.89 & $-3.08^{* *}$ & $-3.50 * *$ & $-2.49 *$ & -2.46 & $-2.82 * *$ \\
\hline 32 & $\mathrm{~L}_{14} \times \mathrm{T}_{1}$ & -1.49 & -1.31 & -0.89 & -1.23 & -1.06 & -2.04 & -1.46 & -1.52 \\
\hline 33 & $\mathrm{~L}_{15} \times \mathrm{T}_{1}$ & -1.38 & $-2.87 *$ & -4.11 & $-2.79 * *$ & -0.61 & $-3.60^{* * *}$ & $-4.57 *$ & $-2.93 * *$ \\
\hline 34 & $\mathrm{~L}_{1} \times \mathrm{T}_{2}$ & $-2.87^{*}$ & -2.02 & -2.20 & $-2.36^{*}$ & $-3.19 * *$ & -2.31 & -1.37 & $-2.29 *$ \\
\hline 35 & $\mathrm{~L}_{2} \times \mathrm{T}_{2}$ & $-3.42 * *$ & $-4.47 * *$ & $-5.09 *$ & $-4.33^{* *}$ & $-3.41 * *$ & $-4.20 * *$ & $-6.26^{* * *}$ & $-4.62 * *$ \\
\hline 36 & $\mathrm{~L}_{3} \times \mathrm{T}_{2}$ & -1.20 & -1.13 & -0.42 & -0.92 & -1.30 & -0.98 & -1.04 & -1.11 \\
\hline
\end{tabular}




\begin{tabular}{|c|c|c|c|c|c|c|c|c|c|}
\hline 37 & $\mathrm{~L}_{4} \times \mathrm{T}_{2}$ & 1.58 & 0.20 & 2.69 & 1.49 & 1.70 & 0.13 & 3.63 & 1.82 \\
\hline 38 & $\mathrm{~L}_{5} \times \mathrm{T}_{2}$ & 1.24 & 1.64 & 1.47 & 1.45 & 0.59 & 1.69 & 1.63 & 1.30 \\
\hline 39 & $\mathrm{~L}_{6} \times \mathrm{T}_{2}$ & -0.87 & -0.91 & -0.42 & -0.73 & -0.64 & -1.31 & -1.15 & -1.03 \\
\hline 40 & $\mathrm{~L}_{7} \times \mathrm{T}_{2}$ & -1.98 & -2.36 & -3.09 & $-2.47 * *$ & -1.86 & -2.09 & -4.04 & $-2.66 * *$ \\
\hline 41 & $\mathrm{~L}_{8} \times \mathrm{T}_{2}$ & $-3.20 * *$ & $-3.47 * *$ & -3.87 & $-3.51^{* *}$ & $-3.86^{* *}$ & $-2.76^{*}$ & -4.26 & $-3.62 * *$ \\
\hline 42 & $\mathrm{~L}_{9} \times \mathrm{T}_{2}$ & $4.58 * *$ & $3.76^{* *}$ & $4.80^{*}$ & $4.38 * *$ & $5.14 * *$ & $4.47 * *$ & $4.63^{*}$ & $4.75^{* *}$ \\
\hline 43 & $\mathrm{~L}_{10} \times \mathrm{T}_{2}$ & -1.20 & -0.02 & -0.87 & -0.70 & -0.97 & -0.09 & -0.26 & -0.44 \\
\hline 44 & $\mathrm{~L}_{11} \times \mathrm{T}_{2}$ & 1.91 & 2.31 & 2.91 & $2.38^{*}$ & 2.14 & 1.80 & 1.96 & $1.97 *$ \\
\hline 45 & $\mathrm{~L}_{12} \times \mathrm{T}_{2}$ & 0.69 & 0.20 & 0.58 & 0.49 & 1.59 & 0.24 & 1.74 & 1.19 \\
\hline 46 & $\mathrm{~L}_{13} \times \mathrm{T}_{2}$ & $3.24 * *$ & $3.64 * *$ & 1.69 & $2.86^{* *}$ & $2.81^{*}$ & 2.24 & 2.85 & $2.63 * *$ \\
\hline 47 & $\mathrm{~L}_{14} \times \mathrm{T}_{2}$ & 0.36 & 0.42 & 0.02 & 0.27 & 0.59 & 0.69 & -0.81 & 0.15 \\
\hline 48 & $\mathrm{~L}_{15} \times \mathrm{T}_{2}$ & 1.13 & 2.20 & 1.80 & 1.71 & 0.70 & $2.47^{*}$ & 2.74 & $1.97 *$ \\
\hline 49 & $\mathrm{~L}_{1} \times \mathrm{T}_{3}$ & 1.24 & $3.11^{*}$ & 4.31 & $2.89 * *$ & 1.03 & $3.36^{* *}$ & $5.05^{*}$ & $3.15^{* *}$ \\
\hline 50 & $\mathrm{~L}_{2} \times \mathrm{T}_{3}$ & $3.02 *$ & $3.67 * *$ & 3.09 & $3.26 * *$ & $3.47 * *$ & $3.13 *$ & 3.16 & $3.26 * *$ \\
\hline 51 & $\mathrm{~L}_{3} \times \mathrm{T}_{3}$ & -1.42 & -1.67 & -2.91 & $-2.00 *$ & -1.41 & -1.31 & -1.61 & -1.45 \\
\hline 52 & $\mathrm{~L}_{4} \times \mathrm{T}_{3}$ & $-2.64 *$ & $-2.67 *$ & -4.13 & $-3.15^{* *}$ & $-2.41^{*}$ & $-2.53^{*}$ & $-4.61 *$ & $-3.19 * *$ \\
\hline 53 & $\mathrm{~L}_{5} \times \mathrm{T}_{3}$ & $-2.64 *$ & $-2.56^{*}$ & -0.69 & $-1.96^{*}$ & -2.19 & $-3.31 * *$ & -2.61 & $-2.71 * *$ \\
\hline 54 & $\mathrm{~L}_{6} \times \mathrm{T}_{3}$ & 1.58 & 1.56 & 1.42 & 1.52 & 1.92 & 1.36 & 1.61 & 1.63 \\
\hline 55 & $\mathrm{~L}_{7} \times \mathrm{T}_{3}$ & 1.47 & 1.44 & 0.09 & 1.00 & 1.70 & 0.91 & 0.72 & 1.11 \\
\hline 56 & $\mathrm{~L}_{8} \times \mathrm{T}_{3}$ & $2.58 *$ & $3.67 * *$ & 4.31 & $3.52^{* *}$ & $3.03 *$ & $3.24 * *$ & 4.16 & $3.48 * *$ \\
\hline 57 & $\mathrm{~L}_{9} \times \mathrm{T}_{3}$ & -1.31 & -1.11 & -2.36 & -1.59 & -1.64 & -1.20 & -1.28 & -1.37 \\
\hline 58 & $\mathrm{~L}_{10} \times \mathrm{T}_{3}$ & 0.24 & -2.22 & -3.36 & -1.78 & -0.41 & -2.09 & -3.50 & $-2.00 *$ \\
\hline 59 & $\mathrm{~L}_{11} \times \mathrm{T}_{3}$ & -1.64 & -1.89 & -2.24 & $-1.93^{*}$ & -1.30 & -2.20 & -1.95 & -1.82 \\
\hline 60 & $\mathrm{~L}_{12} \times \mathrm{T}_{3}$ & -1.87 & -2.33 & -1.91 & $-2.04 *$ & $-2.86^{*}$ & -2.09 & -2.84 & $-2.60 * *$ \\
\hline 61 & $\mathrm{~L}_{13} \times \mathrm{T}_{3}$ & 0.02 & -0.56 & 1.20 & 0.22 & 0.70 & 0.24 & -0.39 & 0.18 \\
\hline 62 & $\mathrm{~L}_{14} \times \mathrm{T}_{3}$ & 1.13 & 0.89 & 0.87 & 0.96 & 0.47 & 1.36 & 2.27 & 1.37 \\
\hline \multirow[t]{11}{*}{63} & $\mathrm{~L}_{15} \times \mathrm{T}_{3}$ & 0.24 & 0.67 & 2.31 & 1.07 & -0.08 & 1.13 & 1.83 & 0.96 \\
\hline & \multicolumn{2}{|c|}{ Standard error } & & & & & & & \\
\hline & $\mathrm{Ti}$ & 0.29 & 0.31 & 0.55 & 0.23 & 0.30 & 0.31 & 0.56 & 0.23 \\
\hline & $\mathrm{Lj}$ & 0.59 & 0.62 & 1.11 & 0.47 & 0.60 & 0.62 & 1.12 & 0.47 \\
\hline & $\mathrm{Sij}$ & 1.18 & 1.24 & 2.22 & 0.93 & 1.19 & 1.24 & 2.23 & 0.94 \\
\hline & Ti-j & 0.36 & 0.38 & 0.68 & 0.29 & 0.36 & 0.38 & 0.68 & 0.29 \\
\hline & $\mathrm{Li}-\mathrm{j}$ & 0.81 & 0.85 & 1.52 & 0.64 & 0.82 & 0.85 & 1.53 & 0.64 \\
\hline & Ti-Lj & 0.63 & 0.66 & 1.18 & 0.50 & 0.63 & 0.66 & 1.18 & 0.50 \\
\hline & STi-Tj & 1.44 & 1.52 & 2.71 & 1.14 & 1.46 & 1.52 & 2.73 & 1.15 \\
\hline & SiL-jL & 1.61 & 1.70 & 3.03 & 1.28 & 1.63 & 1.70 & 3.06 & 1.29 \\
\hline & Sij-kl & 1.65 & 1.74 & 3.11 & 1.31 & 1.67 & 1.74 & 3.13 & 1.32 \\
\hline
\end{tabular}


Table.3 GCA and SCA effects for Antheis - silking interval and Days to 75 per cent brown husk

\begin{tabular}{|c|c|c|c|c|c|c|c|c|c|}
\hline \multirow[t]{2}{*}{ SN } & \multirow[t]{2}{*}{ Genotype } & \multicolumn{4}{|c|}{ Antheis-silking interval } & \multicolumn{4}{|c|}{ Days to 75 per cent brown husk } \\
\hline & & E1 & E2 & E3 & Pool & E1 & E2 & E3 & Pool \\
\hline 1 & $\mathrm{~T}_{1}$ & $-0.10 * *$ & 0.07 & $0.24 * *$ & $0.07 *$ & $-1.47 * *$ & $-1.62 * *$ & -0.86 & $-1.32 * *$ \\
\hline 2 & $\mathrm{~T}_{2}$ & $0.10 * *$ & 0.07 & $-0.50 * *$ & $-0.11 * *$ & -0.62 & -0.62 & -0.93 & $-0.72 *$ \\
\hline 3 & $\mathrm{~T}_{3}$ & -0.01 & $-0.13 * *$ & $0.26^{* *}$ & 0.04 & $2.09 * *$ & $2.24 * *$ & $1.79 *$ & $2.04 * *$ \\
\hline 4 & $\mathrm{~L}_{1}$ & $-0.32 * *$ & 0.11 & $-0.41 *$ & $-0.20 * *$ & 0.36 & 0.31 & 2.67 & 1.11 \\
\hline 5 & $\mathrm{~L}_{2}$ & 0.01 & -0.11 & -0.07 & -0.06 & 0.02 & 0.42 & 0.34 & 0.26 \\
\hline 6 & $\mathrm{~L}_{3}$ & $-0.54 * *$ & 0.00 & $0.37 *$ & -0.06 & 0.80 & -1.69 & 2.34 & 0.48 \\
\hline 7 & $\mathrm{~L}_{4}$ & $0.24 * *$ & $0.22 * *$ & 0.15 & $0.20 * *$ & -1.53 & -1.69 & -2.55 & $-1.92 * *$ \\
\hline 8 & $\mathrm{~L}_{5}$ & 0.01 & 0.11 & 0.26 & 0.13 & $-2.87 * *$ & $-2.13 *$ & $-3.33 *$ & $-2.78 * *$ \\
\hline 9 & $\mathrm{~L}_{6}$ & 0.13 & $-0.44 * *$ & $-0.52 * *$ & $-0.28 * *$ & 1.24 & 1.64 & 0.90 & 1.26 \\
\hline 10 & $\mathrm{~L}_{7}$ & $0.24 * *$ & -0.11 & -0.30 & -0.06 & -0.20 & 0.42 & -1.77 & -0.52 \\
\hline 11 & $\mathrm{~L}_{8}$ & 0.01 & $0.44 * *$ & $1.15^{* *}$ & $0.54 * *$ & 0.36 & 0.76 & 0.90 & 0.67 \\
\hline 12 & $\mathrm{~L}_{9}$ & $-0.21 * *$ & $0.44 * *$ & $-1.07 * *$ & $-0.28 * *$ & 0.69 & -0.47 & 0.67 & 0.30 \\
\hline 13 & $\mathrm{~L}_{10}$ & 0.13 & $0.22 * *$ & 0.15 & $0.17 *$ & $-1.87 *$ & $-2.24 *$ & -1.21 & $-1.78 * *$ \\
\hline 14 & $\mathrm{~L}_{11}$ & 0.13 & $-0.33 * *$ & -0.30 & $-0.17 *$ & 0.47 & 0.98 & -0.44 & 0.34 \\
\hline 15 & $\mathrm{~L}_{12}$ & $0.46^{* *}$ & 0.11 & -0.07 & $0.17 *$ & $-4.42 * *$ & $-2.91 * *$ & $-3.88 * *$ & $-3.74 * *$ \\
\hline 16 & $\mathrm{~L}_{13}$ & $-0.21 * *$ & $-0.44 * *$ & $-0.41 *$ & $-0.35 * *$ & $2.47 * *$ & $3.53 * *$ & 0.34 & $2.11 * *$ \\
\hline 17 & $\mathrm{~L}_{14}$ & 0.13 & -0.11 & $0.59 * *$ & $0.20 * *$ & $2.36 * *$ & 1.42 & 2.34 & $2.04 * *$ \\
\hline 18 & $\mathrm{~L}_{15}$ & $-0.21 * *$ & -0.11 & $0.48 * *$ & 0.05 & $2.13 *$ & 1.64 & 2.67 & $2.15^{* *}$ \\
\hline 19 & $\mathrm{~L}_{1} \times \mathrm{T}_{1}$ & $0.54 * *$ & 0.04 & $-1.57 * *$ & $-0.33 *$ & 1.91 & 0.62 & 1.64 & 1.39 \\
\hline 20 & $\mathrm{~L}_{2} \times \mathrm{T}_{1}$ & $-0.46^{* *}$ & 0.27 & $1.10^{* *}$ & $0.30 *$ & 1.24 & 1.18 & 0.97 & 1.13 \\
\hline 21 & $\mathrm{~L}_{3} \times \mathrm{T}_{1}$ & 0.10 & $-0.51 * *$ & $-0.68 *$ & $-0.37 * *$ & 2.47 & 1.62 & 1.97 & 2.02 \\
\hline 22 & $\mathrm{~L}_{4} \times \mathrm{T}_{1}$ & $-0.35^{*}$ & -0.07 & -0.46 & $-0.29 *$ & -0.20 & 1.29 & 2.19 & 1.09 \\
\hline 23 & $\mathrm{~L}_{5} \times \mathrm{T}_{1}$ & 0.21 & $0.71 * *$ & $1.76^{* *}$ & $0.89 * *$ & 0.47 & 0.73 & -0.03 & 0.39 \\
\hline 24 & $\mathrm{~L}_{6} \times \mathrm{T}_{1}$ & $-0.57 * *$ & $0.60 * *$ & 0.54 & 0.19 & -2.64 & -1.71 & -1.25 & -1.87 \\
\hline 25 & $\mathrm{~L}_{7} \times \mathrm{T}_{1}$ & $-0.35^{*}$ & 0.27 & 0.32 & 0.08 & 1.13 & 0.84 & 1.08 & 1.02 \\
\hline 26 & $\mathrm{~L}_{8} \times \mathrm{T}_{1}$ & 0.21 & -0.29 & 0.54 & 0.15 & 1.24 & 0.84 & 0.75 & 0.95 \\
\hline 27 & $\mathrm{~L}_{9} \times \mathrm{T}_{1}$ & -0.24 & $-0.62 * *$ & $-0.90 * *$ & $-0.59 * *$ & $-4.42 *$ & -1.93 & -2.70 & $-3.02 *$ \\
\hline 28 & $\mathrm{~L}_{10} \times \mathrm{T}_{1}$ & $0.43 * *$ & -0.07 & -0.46 & -0.03 & $5.80 * *$ & 2.51 & 1.53 & $3.28 *$ \\
\hline 29 & $\mathrm{~L}_{11} \times \mathrm{T}_{1}$ & $-0.57 * *$ & $0.82 * *$ & 0.65 & $0.30 *$ & -1.87 & -0.38 & -0.92 & -1.05 \\
\hline 30 & $\mathrm{~L}_{12} \times \mathrm{T}_{1}$ & 0.10 & -0.29 & -0.24 & -0.14 & 0.36 & 1.84 & 0.19 & 0.80 \\
\hline 31 & $\mathrm{~L}_{13} \times \mathrm{T}_{1}$ & -0.24 & $0.60 * *$ & 0.43 & $0.26^{*}$ & $-4.20^{*}$ & -3.27 & -1.03 & $-2.83^{*}$ \\
\hline 32 & $\mathrm{~L}_{14} \times \mathrm{T}_{1}$ & $0.43 * *$ & $-0.73 * *$ & -0.57 & $-0.29 *$ & -0.42 & -2.16 & -0.03 & -0.87 \\
\hline 33 & $\mathrm{~L}_{15} \times \mathrm{T}_{1}$ & $0.76 * *$ & $-0.73 * *$ & -0.46 & -0.14 & -0.87 & -2.04 & -4.36 & -2.42 \\
\hline 34 & $\mathrm{~L}_{1} \times \mathrm{T}_{2}$ & $-0.33^{*}$ & -0.29 & $0.83^{*}$ & 0.07 & -2.93 & -2.38 & -3.96 & $-3.09 *$ \\
\hline 35 & $\mathrm{~L}_{2} \times \mathrm{T}_{2}$ & 0.01 & 0.27 & $-1.17 * *$ & $-0.30 *$ & $-3.60 *$ & -3.49 & -5.30 & $-4.13 * *$ \\
\hline 36 & $\mathrm{~L}_{3} \times \mathrm{T}_{2}$ & -0.10 & 0.16 & -0.61 & -0.19 & -0.04 & 0.29 & -0.96 & -0.24 \\
\hline 37 & $\mathrm{~L}_{4} \times \mathrm{T}_{2}$ & 0.12 & -0.07 & $0.94 * *$ & $0.33^{*}$ & 1.96 & 1.29 & 2.26 & 1.83 \\
\hline 38 & $\mathrm{~L}_{5} \times \mathrm{T}_{2}$ & $-0.66 * *$ & 0.04 & 0.16 & -0.15 & 3.29 & 2.40 & 1.37 & 2.35 \\
\hline
\end{tabular}




\begin{tabular}{|c|c|c|c|c|c|c|c|c|c|}
\hline 39 & $\mathrm{~L}_{6} \times \mathrm{T}_{2}$ & 0.23 & $-0.40^{*}$ & $-0.73^{*}$ & $-0.30 *$ & 1.18 & 0.29 & 0.81 & 0.76 \\
\hline 40 & $\mathrm{~L}_{7} \times \mathrm{T}_{2}$ & 0.12 & 0.27 & $-0.95 * *$ & -0.19 & -3.38 & -3.16 & -3.19 & $-3.24 *$ \\
\hline 41 & $\mathrm{~L}_{8} \times \mathrm{T}_{2}$ & $-0.66^{* *}$ & $0.71 * *$ & -0.39 & -0.11 & $-3.60 *$ & -3.16 & -4.52 & $-3.76 * *$ \\
\hline 42 & $\mathrm{~L}_{9} \times \mathrm{T}_{2}$ & $0.56^{* *}$ & $0.71 * *$ & -0.17 & $0.37 * *$ & $4.73 * *$ & 0.07 & 4.70 & $3.17 *$ \\
\hline 43 & $\mathrm{~L}_{10} \times \mathrm{T}_{2}$ & 0.23 & -0.07 & 0.61 & 0.26 & -2.04 & 0.18 & -0.07 & -0.65 \\
\hline 44 & $\mathrm{~L}_{11} \times \mathrm{T}_{2}$ & 0.23 & $-0.51 * *$ & $-0.95 * *$ & $-0.41 * *$ & 1.96 & 1.62 & 3.81 & 2.46 \\
\hline 45 & $\mathrm{~L}_{12} \times \mathrm{T}_{2}$ & $0.90 * *$ & 0.04 & $1.16^{* *}$ & $0.70 * *$ & -0.16 & 0.51 & -1.74 & -0.46 \\
\hline 46 & $\mathrm{~L}_{13} \times \mathrm{T}_{2}$ & $-0.44 * *$ & $-1.40^{* *}$ & $1.16^{* *}$ & -0.22 & 2.96 & 3.07 & 3.70 & $3.24 *$ \\
\hline 47 & $\mathrm{~L}_{14} \times \mathrm{T}_{2}$ & 0.23 & 0.27 & $-0.84^{*}$ & -0.11 & -1.27 & 1.18 & 2.04 & 0.65 \\
\hline 48 & $\mathrm{~L}_{15} \times \mathrm{T}_{2}$ & $-0.44 * *$ & 0.27 & $0.94 * *$ & 0.26 & 0.96 & 1.29 & 1.04 & 1.09 \\
\hline 49 & $\mathrm{~L}_{1} \times \mathrm{T}_{3}$ & -0.21 & 0.24 & $0.74 *$ & 0.26 & 1.02 & 1.76 & 2.33 & 1.70 \\
\hline 50 & $\mathrm{~L}_{2} \times \mathrm{T}_{3}$ & $0.45^{* *}$ & $-0.53 * *$ & 0.07 & -0.00 & 2.36 & 2.31 & 4.33 & $3.00 *$ \\
\hline 51 & $\mathrm{~L}_{3} \times \mathrm{T}_{3}$ & 0.01 & $0.36^{*}$ & $1.30 * *$ & $0.55 * *$ & -2.42 & -1.91 & -1.01 & -1.78 \\
\hline 52 & $\mathrm{~L}_{4} \times \mathrm{T}_{3}$ & 0.23 & 0.13 & -0.48 & -0.04 & -1.76 & -2.58 & -4.45 & $-2.93 *$ \\
\hline 53 & $\mathrm{~L}_{5} \times \mathrm{T}_{3}$ & $0.45^{* *}$ & $-0.76^{* *}$ & $-1.93 * *$ & $-0.74 * *$ & $-3.76^{*}$ & -3.13 & -1.34 & $-2.74 *$ \\
\hline 54 & $\mathrm{~L}_{6} \times \mathrm{T}_{3}$ & $0.34 *$ & -0.20 & 0.19 & 0.11 & 1.47 & 1.42 & 0.44 & 1.11 \\
\hline 55 & $\mathrm{~L}_{7} \times \mathrm{T}_{3}$ & 0.23 & $-0.53 * *$ & 0.63 & 0.11 & 2.24 & 2.31 & 2.10 & 2.22 \\
\hline 56 & $\mathrm{~L}_{8} \times \mathrm{T}_{3}$ & $0.45^{* *}$ & $-0.42 * *$ & -0.15 & -0.04 & 2.36 & 2.31 & 3.77 & $2.81 *$ \\
\hline 57 & $\mathrm{~L}_{9} \times \mathrm{T}_{3}$ & $-0.33^{*}$ & -0.09 & $1.07 * *$ & 0.22 & -0.31 & 1.87 & -2.01 & -0.15 \\
\hline 58 & $\mathrm{~L}_{10} \times \mathrm{T}_{3}$ & $-0.66 * *$ & 0.13 & -0.15 & -0.22 & $-3.76^{*}$ & -2.69 & -1.45 & $-2.63^{*}$ \\
\hline 59 & $\mathrm{~L}_{11} \times \mathrm{T}_{3}$ & $0.34 *$ & -0.31 & 0.30 & 0.11 & -0.09 & -1.24 & -2.90 & -1.41 \\
\hline 60 & $\mathrm{~L}_{12} \times \mathrm{T}_{3}$ & $-0.99 * *$ & 0.24 & $-0.93 * *$ & $-0.56^{* *}$ & -0.20 & -2.36 & 1.55 & -0.34 \\
\hline 61 & $\mathrm{~L}_{13} \times \mathrm{T}_{3}$ & $0.67 * *$ & $0.80 * *$ & $-1.59 * *$ & -0.04 & 1.24 & 0.20 & -2.67 & -0.41 \\
\hline 62 & $\mathrm{~L}_{14} \times \mathrm{T}_{3}$ & $-0.66^{* *}$ & $0.47 * *$ & $1.41 * *$ & $0.40 * *$ & 1.69 & 0.98 & -2.01 & 0.22 \\
\hline \multirow[t]{11}{*}{63} & $\mathrm{~L}_{15} \times \mathrm{T}_{3}$ & $-0.33^{*}$ & $0.47 * *$ & -0.48 & -0.11 & -0.09 & 0.76 & 3.33 & 1.33 \\
\hline & \multicolumn{2}{|c|}{ Standard error } & & & & & & & \\
\hline & $\mathrm{Ti}$ & 0.04 & 0.04 & 0.08 & 0.03 & 0.44 & 0.45 & 0.74 & 0.32 \\
\hline & $\mathrm{Lj}$ & 0.07 & 0.08 & 0.17 & 0.07 & 0.89 & 0.89 & 1.48 & 0.65 \\
\hline & $\mathrm{Sij}$ & 0.14 & 0.16 & 0.33 & 0.13 & 1.77 & 1.79 & 2.97 & 1.30 \\
\hline & $\mathrm{Ti}-\mathrm{j}$ & 0.04 & 0.05 & 0.10 & 0.04 & 0.54 & 0.55 & 0.91 & 0.40 \\
\hline & $\mathrm{Li}-\mathrm{j}$ & 0.10 & 0.11 & 0.23 & 0.09 & 1.21 & 1.23 & 2.03 & 0.89 \\
\hline & Ti-Lj & 0.08 & 0.09 & 0.18 & 0.07 & 0.94 & 0.95 & 1.57 & 0.69 \\
\hline & STi-Tj & 0.18 & 0.20 & 0.41 & 0.16 & 2.17 & 2.19 & 3.63 & 1.59 \\
\hline & SiL-jL & 0.20 & 0.22 & 0.46 & 0.18 & 2.43 & 2.45 & 4.06 & 1.78 \\
\hline & Sij-kl & 0.20 & 0.23 & 0.47 & 0.19 & 2.49 & 2.51 & 4.16 & 1.82 \\
\hline
\end{tabular}

*** Significantly deviating from zero for $5 \%$ and $1 \%$ level of significance 
Table.4 GCA and SCA effects for plant height $(\mathrm{cm})$ and ear height $(\mathrm{cm})$

\begin{tabular}{|c|c|c|c|c|c|c|c|c|c|}
\hline SN & Genotype & & Plant he & ght (cm) & & & Ear he & ght (cm) & \\
\hline & & E1 & E2 & E3 & Pool & E1 & E2 & E3 & Pool \\
\hline 1 & $\mathrm{~T}_{1}$ & $-7.37 * *$ & $-5.80^{* *}$ & $-6.82 * *$ & $-6.66^{* *}$ & $-10.93 * *$ & $-9.30 * *$ & $-10.31 * *$ & $-10.18^{* *}$ \\
\hline 2 & $\mathrm{~T}_{2}$ & 0.28 & -0.80 & -0.21 & -0.24 & $4.25^{* *}$ & $3.81 * *$ & $4.47 * *$ & $4.18^{* *}$ \\
\hline 3 & $\mathrm{~T}_{3}$ & $7.08^{* *}$ & $6.60 * *$ & $7.03 * *$ & $6.90 * *$ & $6.67 * *$ & $5.50 * *$ & $5.84 * *$ & $6.00^{* * *}$ \\
\hline 4 & $\mathrm{~L}_{1}$ & 4.20 & -1.64 & -1.41 & 0.38 & $-7.61^{* *}$ & $-8.90 * *$ & $-8.89 * *$ & $-8.47 * *$ \\
\hline 5 & $\mathrm{~L}_{2}$ & 0.61 & 0.55 & 7.32 & 2.82 & $-4.50 * *$ & $-5.68 * *$ & $-5.44 * *$ & $-5.21 * *$ \\
\hline 6 & $\mathrm{~L}_{3}$ & $-8.51 *$ & $-14.35^{* *}$ & 2.46 & $-6.80 * *$ & $-7.50 * *$ & $-7.13^{* *}$ & $-7.44 * *$ & $-7.36^{* *}$ \\
\hline 7 & $\mathrm{~L}_{4}$ & 1.94 & 0.27 & $9.55^{*}$ & 3.92 & $-3.95^{*}$ & -3.24 & -3.22 & $-3.47 * *$ \\
\hline 8 & $\mathrm{~L}_{5}$ & $-9.54 *$ & -4.70 & $-10.06^{*}$ & $-8.10 * *$ & 1.50 & 3.65 & 1.00 & $2.05^{*}$ \\
\hline 9 & $\mathrm{~L}_{6}$ & -1.37 & 5.37 & -0.71 & 1.10 & 2.27 & 2.76 & $3.89 *$ & $2.98 * *$ \\
\hline 10 & $\mathrm{~L}_{7}$ & 2.96 & 5.38 & $-11.71 *$ & -1.12 & 2.83 & 1.10 & 0.44 & 1.46 \\
\hline 11 & $\mathrm{~L}_{8}$ & -5.06 & 0.76 & 0.70 & -1.20 & 0.61 & 0.99 & 1.11 & 0.90 \\
\hline 12 & $\mathrm{~L}_{9}$ & -1.68 & 5.92 & $11.00 *$ & $5.08^{*}$ & $4.05^{* *}$ & $4.54^{*}$ & $5.33 * *$ & $4.64^{* * *}$ \\
\hline 13 & $\mathrm{~L}_{10}$ & -6.60 & $-11.94 * *$ & $-11.05^{*}$ & $-9.86^{* *}$ & $-6.95^{* *}$ & $-5.79 * *$ & -3.44 & $-5.40 * *$ \\
\hline 14 & $\mathrm{~L}_{11}$ & 7.63 & -1.73 & $9.71^{*}$ & $5.20^{*}$ & $3.83^{*}$ & 3.21 & $4.44^{*}$ & $3.83^{* *}$ \\
\hline 15 & $\mathrm{~L}_{12}$ & -2.61 & 2.65 & 6.82 & 2.29 & $5.72 * *$ & 2.99 & 1.89 & $3.53^{* *}$ \\
\hline 16 & $\mathrm{~L}_{13}$ & 5.60 & 2.97 & $-21.09 * *$ & -4.17 & 2.05 & 2.87 & 2.00 & $2.31^{*}$ \\
\hline 17 & $\mathrm{~L}_{14}$ & 8.15 & -1.94 & 7.49 & 4.56 & $6.16^{* *}$ & $6.54 * *$ & $5.67 * *$ & $6.12^{* * *}$ \\
\hline 18 & $\mathrm{~L}_{15}$ & 4.29 & $12.44 * *$ & 1.00 & $5.91^{*}$ & 1.50 & 2.10 & 2.67 & $2.09 *$ \\
\hline 19 & $\mathrm{~L}_{1} \times \mathrm{T}_{1}$ & 9.80 & 8.29 & -10.22 & 2.62 & $14.48^{* *}$ & $12.53^{* *}$ & $10.31 * *$ & $12.44^{* *}$ \\
\hline 20 & $\mathrm{~L}_{2} \times \mathrm{T}_{1}$ & 5.62 & 1.58 & 8.32 & 5.18 & 4.37 & 4.30 & 4.87 & $4.51 *$ \\
\hline 21 & $\mathrm{~L}_{3} \times \mathrm{T}_{1}$ & 9.35 & 15.91 & 16.18 & $13.81 * *$ & $10.37^{* *}$ & $10.41^{*}$ & 5.87 & $8.88^{* *}$ \\
\hline 22 & $\mathrm{~L}_{4} \times \mathrm{T}_{1}$ & 12.22 & 10.43 & 12.67 & $11.77^{*}$ & $6.15^{*}$ & 4.19 & 5.64 & $5.33^{*}$ \\
\hline 23 & $\mathrm{~L}_{5} \times \mathrm{T}_{1}$ & -1.92 & -4.40 & 15.56 & 3.08 & -2.30 & -6.03 & -0.91 & -3.08 \\
\hline 24 & $\mathrm{~L}_{6} \times \mathrm{T}_{1}$ & -3.07 & -8.18 & -1.95 & -4.40 & -3.41 & -4.14 & -0.80 & -2.78 \\
\hline 25 & $\mathrm{~L}_{7} \times \mathrm{T}_{1}$ & 8.61 & 5.00 & -2.79 & 3.61 & 5.04 & 3.19 & 1.31 & 3.18 \\
\hline 26 & $\mathrm{~L}_{8} \times \mathrm{T}_{1}$ & -10.78 & 9.84 & -11.50 & -4.15 & -3.41 & -1.70 & -3.69 & -2.93 \\
\hline 27 & $\mathrm{~L}_{9} \times \mathrm{T}_{1}$ & $-22.16^{* *}$ & -3.67 & -12.20 & $-12.68^{*}$ & $-11.85 * *$ & $-9.92 *$ & $-11.58^{* *}$ & $-11.12^{* *}$ \\
\hline 28 & $\mathrm{~L}_{10} \times \mathrm{T}_{1}$ & 6.21 & -0.50 & -6.29 & -0.19 & 0.81 & 2.41 & 2.87 & 2.03 \\
\hline 29 & $\mathrm{~L}_{11} \times \mathrm{T}_{1}$ & -9.21 & $-17.40 *$ & 6.61 & -6.67 & $-6.63^{*}$ & -5.92 & -6.02 & $-6.19 * *$ \\
\hline 30 & $\mathrm{~L}_{12} \times \mathrm{T}_{1}$ & -7.80 & $-20.45^{*}$ & -5.52 & $-11.26^{*}$ & $-12.85^{* *}$ & -7.36 & $-7.47 *$ & $-9.23 * *$ \\
\hline 31 & $\mathrm{~L}_{13} \times \mathrm{T}_{1}$ & 6.07 & -3.88 & 3.39 & 1.86 & -1.52 & -1.59 & -0.58 & -1.23 \\
\hline 32 & $\mathrm{~L}_{14} \times \mathrm{T}_{1}$ & 6.85 & 7.22 & -6.35 & 2.57 & 2.70 & 0.41 & 3.42 & 2.18 \\
\hline 33 & $\mathrm{~L}_{15} \times \mathrm{T}_{1}$ & -9.81 & 0.21 & -5.90 & -5.17 & -1.96 & -0.81 & -3.24 & -2.00 \\
\hline 34 & $\mathrm{~L}_{1} \times \mathrm{T}_{2}$ & -3.93 & -1.43 & 3.13 & -0.74 & -3.36 & -4.59 & -4.47 & $-4.14^{*}$ \\
\hline 35 & $\mathrm{~L}_{2} \times \mathrm{T}_{2}$ & 13.41 & $17.16^{*}$ & 10.69 & $13.75^{* *}$ & $6.86^{*}$ & 6.53 & 3.76 & $5.71^{* *}$ \\
\hline 36 & $\mathrm{~L}_{3} \times \mathrm{T}_{2}$ & 10.09 & 0.71 & 5.70 & 5.50 & 1.19 & -0.36 & 2.42 & 1.08 \\
\hline
\end{tabular}




\begin{tabular}{|c|c|c|c|c|c|c|c|c|c|}
\hline 37 & $\mathrm{~L}_{4} \times \mathrm{T}_{2}$ & 8.00 & 14.87 & 6.70 & $9.86^{*}$ & 3.30 & 3.75 & 2.87 & 3.31 \\
\hline 38 & $\mathrm{~L}_{5} \times \mathrm{T}_{2}$ & -9.00 & $-21.65 * *$ & 7.31 & -7.78 & $-12.81 * *$ & $-8.14^{*}$ & $-11.36 * *$ & $-10.77 * *$ \\
\hline 39 & $\mathrm{~L}_{6} \times \mathrm{T}_{2}$ & -14.80 & 2.85 & $-31.73 * *$ & $-14.56^{* *}$ & -0.25 & 1.41 & 3.76 & 1.64 \\
\hline 40 & $\mathrm{~L}_{7} \times \mathrm{T}_{2}$ & -10.37 & -4.80 & 13.71 & -0.49 & -3.81 & -4.59 & -3.13 & -3.84 \\
\hline 41 & $\mathrm{~L}_{8} \times \mathrm{T}_{2}$ & 3.34 & -6.38 & 3.23 & 0.06 & 1.41 & 1.53 & 1.53 & 1.49 \\
\hline 42 & $\mathrm{~L}_{9} \times \mathrm{T}_{2}$ & 11.79 & -2.66 & 3.42 & 4.19 & 3.97 & 1.64 & 4.64 & 3.42 \\
\hline 43 & $\mathrm{~L}_{10} \times \mathrm{T}_{2}$ & 0.27 & 1.03 & -7.40 & -2.03 & 0.97 & -1.03 & -0.91 & -0.32 \\
\hline 44 & $\mathrm{~L}_{11} \times \mathrm{T}_{2}$ & 1.22 & 9.15 & 0.64 & 3.67 & $8.19 * *$ & $8.30 *$ & $8.20 *$ & $8.23 * *$ \\
\hline 45 & $\mathrm{~L}_{12} \times \mathrm{T}_{2}$ & 15.13 & $17.45^{*}$ & 5.53 & $12.70 *$ & $8.30 * *$ & $10.86 * *$ & $9.76 * *$ & $9.64 * *$ \\
\hline 46 & $\mathrm{~L}_{13} \times \mathrm{T}_{2}$ & -2.26 & -0.21 & $-20.19 *$ & -7.55 & 1.64 & 1.64 & 1.31 & 1.53 \\
\hline 47 & $\mathrm{~L}_{14} \times \mathrm{T}_{2}$ & $-21.09 *$ & $-24.09 * *$ & -10.47 & $-18.55^{* *}$ & $-12.14 * *$ & $-13.36 * *$ & $-13.02 * *$ & $-12.84 * *$ \\
\hline 48 & $\mathrm{~L}_{15} \times \mathrm{T}_{2}$ & -1.77 & -2.01 & 9.72 & 1.98 & -3.47 & -3.59 & -5.36 & $-4.14^{*}$ \\
\hline 49 & $\mathrm{~L}_{1} \times \mathrm{T}_{3}$ & -5.87 & -6.86 & 7.09 & -1.88 & $-11.12 * *$ & -7.94 & -5.84 & $-8.30 * *$ \\
\hline 50 & $\mathrm{~L}_{2} \times \mathrm{T}_{3}$ & $-19.03^{*}$ & $-18.75^{*}$ & $-19.01 *$ & $-18.93 * *$ & $-11.23 * *$ & $-10.83 * *$ & $-8.62 *$ & $-10.23^{* *}$ \\
\hline 51 & $\mathrm{~L}_{3} \times \mathrm{T}_{3}$ & $-19.44^{*}$ & $-16.62 *$ & $-21.88 *$ & $-19.31 * *$ & $-11.56^{* *}$ & $-10.05^{*}$ & $-8.29 *$ & $-9.97 * *$ \\
\hline 52 & $\mathrm{~L}_{4} \times \mathrm{T}_{3}$ & $-20.23^{*}$ & $-25.30 * *$ & $-19.37 *$ & $-21.63 * *$ & $-9.45^{* *}$ & -7.94 & $-8.51 *$ & $-8.63 * *$ \\
\hline 53 & $\mathrm{~L}_{5} \times \mathrm{T}_{3}$ & 10.92 & $26.05 * *$ & $-22.87 *$ & 4.70 & $15.10 * *$ & $14.17 * *$ & $12.27 * *$ & $13.85 * *$ \\
\hline 54 & $\mathrm{~L}_{6} \times \mathrm{T}_{3}$ & $17.88^{*}$ & 5.32 & $33.69 * *$ & $18.96 * *$ & 3.66 & 2.73 & -2.96 & 1.14 \\
\hline 55 & $\mathrm{~L}_{7} \times \mathrm{T}_{3}$ & 1.76 & -0.20 & -10.92 & -3.12 & -1.23 & 1.39 & 1.82 & 0.66 \\
\hline 56 & $\mathrm{~L}_{8} \times \mathrm{T}_{3}$ & 7.44 & -3.46 & 8.28 & 4.09 & 1.99 & 0.17 & 2.16 & 1.44 \\
\hline 57 & $\mathrm{~L}_{9} \times \mathrm{T}_{3}$ & 10.37 & 6.33 & 8.78 & 8.49 & $7.88^{*}$ & $8.28 *$ & 6.93 & $7.70 * *$ \\
\hline 58 & $\mathrm{~L}_{10} \times \mathrm{T}_{3}$ & -6.48 & -0.53 & 13.69 & 2.23 & -1.79 & -1.39 & -1.96 & -1.71 \\
\hline 59 & $\mathrm{~L}_{11} \times \mathrm{T}_{3}$ & 7.99 & 8.25 & -7.25 & 3.00 & -1.56 & -2.39 & -2.18 & -2.04 \\
\hline 60 & $\mathrm{~L}_{12} \times \mathrm{T}_{3}$ & -7.33 & 3.01 & -0.01 & -1.45 & 4.55 & -3.50 & -2.29 & -0.41 \\
\hline 61 & $\mathrm{~L}_{13} \times \mathrm{T}_{3}$ & -3.80 & 4.09 & 16.80 & 5.69 & -0.12 & -0.05 & -0.73 & -0.30 \\
\hline 62 & $\mathrm{~L}_{14} \times \mathrm{T}_{3}$ & 14.24 & $16.87^{*}$ & 16.82 & $15.98 * *$ & $9.44 * *$ & $12.95 * *$ & $9.60 * *$ & $10.66 * *$ \\
\hline \multirow[t]{11}{*}{63} & $\mathrm{~L}_{15} \times \mathrm{T}_{3}$ & 11.58 & 1.80 & -3.82 & 3.19 & 5.44 & 4.39 & $8.60 *$ & $6.14 * *$ \\
\hline & \multicolumn{2}{|c|}{ Standard error } & & & & & & & \\
\hline & $\mathrm{Ti}$ & 2.07 & 2.02 & 2.35 & 1.24 & 0.77 & 1.01 & 0.90 & 0.52 \\
\hline & $\mathrm{Lj}$ & 4.14 & 4.04 & 4.69 & 2.48 & 1.54 & 2.01 & 1.79 & 1.03 \\
\hline & Sij & 8.28 & 8.09 & 9.38 & 4.97 & 3.08 & 4.02 & 3.58 & 2.07 \\
\hline & $\mathrm{Ti}-\mathrm{j}$ & 2.53 & 2.48 & 2.87 & 1.52 & 0.94 & 1.23 & 1.10 & 0.63 \\
\hline & $\mathrm{Li}-\mathrm{j}$ & 5.67 & 5.54 & 6.42 & 3.40 & 2.11 & 2.75 & 2.45 & 1.42 \\
\hline & Ti-Lj & 4.39 & 4.29 & 4.98 & 2.63 & 1.64 & 2.13 & 1.90 & 1.10 \\
\hline & $\mathrm{STi}-\mathrm{Tj}$ & 10.14 & 9.90 & 11.49 & 6.08 & 3.78 & 4.92 & 4.39 & 2.53 \\
\hline & SiL-jL & 11.33 & 11.07 & 12.85 & 6.80 & 4.22 & 5.51 & 4.91 & 2.83 \\
\hline & Sij-kl & 11.61 & 11.35 & 13.16 & 6.97 & 4.33 & 5.64 & 5.03 & 2.90 \\
\hline
\end{tabular}

*,** Significantly deviating from zero for $5 \%$ and $1 \%$ level of significance 
Table.5 GCA and SCA effects for cob length $(\mathrm{cm})$ and number of grain rows/ear

\begin{tabular}{|c|c|c|c|c|c|c|c|c|c|}
\hline SN & Genotype & & Cob Len & th (cm) & & $\mathrm{Nu}$ & iber of $G$ & ain row & \\
\hline & & E1 & E2 & E3 & Pool & E1 & E2 & E3 & Pool \\
\hline 1 & $\mathrm{~T}_{1}$ & $0.33^{*}$ & $0.50 *$ & $0.45^{* *}$ & $0.43^{* *}$ & $-0.28^{*}$ & $-0.57 * *$ & $-0.42^{*}$ & $-0.42^{* *}$ \\
\hline 2 & $\mathrm{~T}_{2}$ & -0.11 & $-0.59 * *$ & 0.12 & -0.19 & 0.19 & 0.04 & 0.31 & 0.18 \\
\hline 3 & $\mathrm{~T}_{3}$ & -0.21 & 0.09 & $-0.58 * *$ & $-0.23^{*}$ & 0.09 & $0.52 * *$ & 0.11 & $0.24 * *$ \\
\hline 4 & $\mathrm{~L}_{1}$ & -0.64 & $-0.91 *$ & 0.24 & $-0.44^{*}$ & $-0.80 * *$ & $-1.35^{* *}$ & $-1.28 * *$ & $-1.14 * *$ \\
\hline 5 & $\mathrm{~L}_{2}$ & $1.03 * *$ & $1.07 *$ & 0.17 & $0.75^{* *}$ & -0.27 & 0.65 & 0.33 & 0.24 \\
\hline 6 & $\mathrm{~L}_{3}$ & $0.68 *$ & -0.51 & $0.89 * *$ & 0.36 & -0.52 & $-0.78^{*}$ & -0.53 & $-0.61 * *$ \\
\hline 7 & $\mathrm{~L}_{4}$ & 0.12 & -0.44 & 0.13 & -0.07 & -0.30 & -0.18 & -0.13 & -0.20 \\
\hline 8 & $\mathrm{~L}_{5}$ & -0.42 & $0.89^{*}$ & 0.01 & 0.16 & 0.21 & 0.14 & 0.22 & 0.19 \\
\hline 9 & $\mathrm{~L}_{6}$ & $0.67 *$ & -0.49 & 0.01 & 0.06 & -0.20 & -0.12 & -0.15 & -0.15 \\
\hline 10 & $\mathrm{~L}_{7}$ & 0.36 & -0.16 & $0.82^{* *}$ & 0.34 & 0.28 & 0.25 & 0.10 & 0.21 \\
\hline 11 & $\mathrm{~L}_{8}$ & -0.03 & 0.25 & $0.68^{*}$ & 0.30 & 0.28 & -0.46 & -0.07 & -0.08 \\
\hline 12 & $\mathrm{~L}_{9}$ & 0.57 & -0.13 & $-0.72 *$ & -0.09 & $0.85^{* *}$ & 0.23 & 0.34 & $0.47 *$ \\
\hline 13 & $\mathrm{~L}_{10}$ & 0.17 & -0.11 & $1.14 * *$ & $0.40^{*}$ & $1.16^{* *}$ & $1.28 * *$ & $0.89^{*}$ & $1.11 * *$ \\
\hline 14 & $\mathrm{~L}_{11}$ & $-0.89 * *$ & -0.06 & $-1.21 * *$ & $-0.72 * *$ & -0.19 & -0.24 & -0.20 & -0.21 \\
\hline 15 & $\mathrm{~L}_{12}$ & $0.91 * *$ & $2.77 * *$ & $1.30 * *$ & $1.66 * *$ & $1.60 * *$ & $1.58 * *$ & $1.69 * *$ & $1.62 * *$ \\
\hline 16 & $\mathrm{~L}_{13}$ & $-0.96 * *$ & $-1.43^{* *}$ & $-0.93 * *$ & $-1.11 * *$ & -0.50 & -0.36 & -0.31 & $-0.39 *$ \\
\hline 17 & $\mathrm{~L}_{14}$ & $-0.83^{*}$ & -0.31 & $-1.01 * *$ & $-0.72 * *$ & -0.24 & 0.06 & 0.19 & 0.00 \\
\hline 18 & $\mathrm{~L}_{15}$ & $-0.75^{*}$ & -0.43 & $-1.52 * *$ & $-0.90 * *$ & $-1.34 * *$ & $-0.70 *$ & $-1.11 * *$ & $-1.05 * *$ \\
\hline 19 & $\mathrm{~L}_{1} \times \mathrm{T}_{1}$ & 0.11 & 0.90 & $-1.33^{*}$ & -0.11 & -0.26 & -0.48 & -0.18 & -0.31 \\
\hline 20 & $\mathrm{~L}_{2} \times \mathrm{T}_{1}$ & -0.20 & -1.01 & -0.67 & -0.63 & -0.32 & 1.27 & 0.26 & 0.41 \\
\hline 21 & $\mathrm{~L}_{3} \times \mathrm{T}_{1}$ & $-1.54 *$ & -0.70 & 0.43 & -0.60 & 0.66 & 0.47 & 1.35 & $0.83^{*}$ \\
\hline 22 & $\mathrm{~L}_{4} \times \mathrm{T}_{1}$ & 1.01 & $1.86^{*}$ & $-1.25^{*}$ & 0.54 & 0.25 & 1.23 & -0.20 & 0.43 \\
\hline 23 & $\mathrm{~L}_{5} \times \mathrm{T}_{1}$ & 0.59 & -0.06 & $2.69 * *$ & $1.07 * *$ & 0.51 & 0.64 & 0.69 & 0.61 \\
\hline 24 & $\mathrm{~L}_{6} \times \mathrm{T}_{1}$ & 0.54 & -0.33 & 0.67 & 0.30 & $1.16^{*}$ & 1.12 & 0.92 & $1.07 * *$ \\
\hline 25 & $\mathrm{~L}_{7} \times \mathrm{T}_{1}$ & -0.61 & -0.72 & 0.36 & -0.32 & -0.35 & -0.21 & -0.47 & -0.35 \\
\hline 26 & $\mathrm{~L}_{8} \times \mathrm{T}_{1}$ & -0.42 & -1.43 & -0.63 & $-0.83^{*}$ & $-1.38^{*}$ & -0.58 & -1.33 & $-1.09 * *$ \\
\hline 27 & $\mathrm{~L}_{9} \times \mathrm{T}_{1}$ & 1.12 & $1.76^{*}$ & -0.73 & 0.72 & $1.98^{* *}$ & 0.69 & 1.28 & $1.32^{* * *}$ \\
\hline 28 & $\mathrm{~L}_{10} \times \mathrm{T}_{1}$ & $-1.38^{*}$ & $-1.83^{*}$ & $-1.60 * *$ & $-1.60 * *$ & -0.63 & -0.30 & 0.16 & -0.26 \\
\hline 29 & $\mathrm{~L}_{11} \times \mathrm{T}_{1}$ & -0.97 & -0.42 & -0.95 & -0.78 & 0.05 & -0.23 & -0.38 & -0.18 \\
\hline 30 & $\mathrm{~L}_{12} \times \mathrm{T}_{1}$ & -0.69 & 0.25 & 0.96 & 0.17 & -0.74 & -1.23 & -0.88 & $-0.95^{*}$ \\
\hline 31 & $\mathrm{~L}_{13} \times \mathrm{T}_{1}$ & 0.96 & 0.22 & 1.07 & 0.75 & -0.20 & -1.31 & 0.07 & -0.48 \\
\hline 32 & $\mathrm{~L}_{14} \times \mathrm{T}_{1}$ & 0.97 & $1.69 *$ & $2.27 * *$ & $1.64 * *$ & -0.04 & -0.29 & -0.40 & -0.24 \\
\hline 33 & $\mathrm{~L}_{15} \times \mathrm{T}_{1}$ & 0.50 & -0.19 & $-1.29^{*}$ & -0.33 & -0.72 & -0.78 & -0.89 & $-0.79 *$ \\
\hline 34 & $\mathrm{~L}_{1} \times \mathrm{T}_{2}$ & 0.18 & 0.40 & 1.08 & 0.55 & 0.58 & 0.31 & 0.38 & 0.42 \\
\hline 35 & $\mathrm{~L}_{2} \times \mathrm{T}_{2}$ & 0.05 & -0.20 & $1.18 *$ & 0.34 & 0.87 & -0.72 & 0.58 & 0.25 \\
\hline 36 & $\mathrm{~L}_{3} \times \mathrm{T}_{2}$ & 0.06 & 0.32 & -0.03 & 0.12 & -0.09 & -0.54 & -0.54 & -0.39 \\
\hline
\end{tabular}




\begin{tabular}{|c|c|c|c|c|c|c|c|c|c|}
\hline 37 & $\mathrm{~L}_{4} \times \mathrm{T}_{2}$ & -0.46 & -0.46 & $1.49 * *$ & 0.19 & -0.01 & -1.26 & 0.39 & -0.29 \\
\hline 38 & $\mathrm{~L}_{5} \times \mathrm{T}_{2}$ & -1.09 & $-1.76^{*}$ & $-2.63^{* *}$ & $-1.83^{* *}$ & $-2.03^{* *}$ & -0.70 & $-2.13^{* *}$ & $-1.62 * *$ \\
\hline 39 & $\mathrm{~L}_{6} \times \mathrm{T}_{2}$ & 0.16 & -0.22 & $-1.16^{*}$ & -0.41 & -0.71 & -0.96 & -1.07 & $-0.91 *$ \\
\hline 40 & $\mathrm{~L}_{7} \times \mathrm{T}_{2}$ & $1.31^{*}$ & $1.67^{*}$ & $1.31^{*}$ & $1.43^{* *}$ & $2.07 * *$ & $1.81 * *$ & $2.26 * *$ & $2.05^{* *}$ \\
\hline 41 & $\mathrm{~L}_{8} \times \mathrm{T}_{2}$ & $1.66^{*}$ & $2.47 * *$ & $2.78^{* * *}$ & $2.30^{* * *}$ & $1.23^{*}$ & 0.76 & $2.19 * *$ & $1.39 * *$ \\
\hline 42 & $\mathrm{~L}_{9} \times \mathrm{T}_{2}$ & 0.02 & -0.56 & $-1.33^{*}$ & -0.62 & -0.50 & -0.23 & -0.17 & -0.30 \\
\hline 43 & $\mathrm{~L}_{10} \times \mathrm{T}_{2}$ & -0.06 & -0.52 & -1.09 & -0.56 & $-1.67 * *$ & -0.83 & $-1.71 *$ & $-1.40 * *$ \\
\hline 44 & $\mathrm{~L}_{11} \times \mathrm{T}_{2}$ & 0.36 & 0.46 & 0.45 & 0.42 & 0.15 & -0.01 & 1.07 & 0.41 \\
\hline 45 & $\mathrm{~L}_{12} \times \mathrm{T}_{2}$ & -0.56 & 0.40 & -0.68 & -0.28 & -0.13 & -0.03 & -0.47 & -0.21 \\
\hline 46 & $\mathrm{~L}_{13} \times \mathrm{T}_{2}$ & -0.47 & -0.00 & -0.45 & -0.30 & 0.23 & 1.10 & -0.45 & 0.30 \\
\hline 47 & $\mathrm{~L}_{14} \times \mathrm{T}_{2}$ & -1.29 & $-1.81 *$ & $-2.48 * *$ & $-1.86^{* *}$ & -0.76 & 0.20 & -0.59 & -0.38 \\
\hline 48 & $\mathrm{~L}_{15} \times \mathrm{T}_{2}$ & 0.13 & -0.19 & $1.55^{* * *}$ & 0.50 & 0.77 & 1.09 & 0.25 & 0.70 \\
\hline 49 & $\mathrm{~L}_{1} \times \mathrm{T}_{3}$ & -0.29 & -1.29 & 0.25 & -0.45 & -0.32 & 0.17 & -0.20 & -0.12 \\
\hline 50 & $\mathrm{~L}_{2} \times \mathrm{T}_{3}$ & 0.15 & 1.21 & -0.51 & 0.28 & -0.55 & -0.56 & -0.84 & -0.65 \\
\hline 51 & $\mathrm{~L}_{3} \times \mathrm{T}_{3}$ & $1.48^{*}$ & 0.38 & -0.40 & 0.49 & -0.57 & 0.07 & -0.81 & -0.44 \\
\hline 52 & $\mathrm{~L}_{4} \times \mathrm{T}_{3}$ & -0.54 & -1.40 & -0.23 & -0.73 & -0.24 & 0.03 & -0.19 & -0.13 \\
\hline 53 & $\mathrm{~L}_{5} \times \mathrm{T}_{3}$ & 0.51 & $1.82^{*}$ & -0.06 & 0.75 & $1.52^{* *}$ & 0.07 & $1.44^{*}$ & $1.01 * *$ \\
\hline 54 & $\mathrm{~L}_{6} \times \mathrm{T}_{3}$ & -0.70 & 0.55 & 0.49 & 0.11 & -0.46 & -0.15 & 0.15 & -0.15 \\
\hline 55 & $\mathrm{~L}_{7} \times \mathrm{T}_{3}$ & -0.70 & -0.96 & $-1.67 * *$ & $-1.11^{* *}$ & $-1.72^{* *}$ & $-1.60 *$ & $-1.78 *$ & $-1.70 * *$ \\
\hline 56 & $\mathrm{~L}_{8} \times \mathrm{T}_{3}$ & -1.24 & -1.04 & $-2.15^{* *}$ & $-1.48^{* *}$ & 0.15 & -0.18 & -0.86 & -0.30 \\
\hline 57 & $\mathrm{~L}_{9} \times \mathrm{T}_{3}$ & -1.14 & -1.20 & $2.05^{* *}$ & -0.10 & $-1.48^{* *}$ & -0.46 & -1.11 & $-1.02 * *$ \\
\hline 58 & $\mathrm{~L}_{10} \times \mathrm{T}_{3}$ & $1.44^{*}$ & $2.35^{* * *}$ & $2.68^{* *}$ & $2.16^{* * *}$ & $2.30^{* * *}$ & 1.13 & $1.54 *$ & $1.66^{* *}$ \\
\hline 59 & $\mathrm{~L}_{11} \times \mathrm{T}_{3}$ & 0.61 & -0.04 & 0.50 & 0.36 & -0.20 & 0.23 & -0.69 & -0.22 \\
\hline 60 & $\mathrm{~L}_{12} \times \mathrm{T}_{3}$ & 1.25 & -0.65 & -0.28 & 0.11 & 0.87 & 1.27 & 1.35 & $1.16^{* * *}$ \\
\hline 61 & $\mathrm{~L}_{13} \times \mathrm{T}_{3}$ & -0.50 & -0.22 & -0.63 & -0.45 & -0.04 & 0.21 & 0.38 & 0.18 \\
\hline 62 & $\mathrm{~L}_{14} \times \mathrm{T}_{3}$ & 0.32 & 0.12 & 0.20 & 0.21 & 0.80 & 0.09 & 0.99 & 0.63 \\
\hline \multirow[t]{11}{*}{63} & $\mathrm{~L}_{15} \times \mathrm{T}_{3}$ & -0.64 & 0.38 & -0.26 & -0.17 & -0.05 & -0.31 & 0.63 & 0.09 \\
\hline & \multicolumn{2}{|c|}{ Standard error } & & & & & & & \\
\hline & $\mathrm{Ti}$ & 0.17 & 0.21 & 0.14 & 0.10 & 0.13 & 0.17 & 0.17 & 0.09 \\
\hline & $\mathrm{Lj}$ & 0.33 & 0.41 & 0.28 & 0.20 & 0.27 & 0.35 & 0.35 & 0.19 \\
\hline & Sij & 0.66 & 0.82 & 0.56 & 0.40 & 0.54 & 0.69 & 0.69 & 0.37 \\
\hline & Ti-j & 0.20 & 0.25 & 0.17 & 0.12 & 0.16 & 0.21 & 0.21 & 0.11 \\
\hline & $\mathrm{Li}-\mathrm{j}$ & 0.45 & 0.56 & 0.38 & 0.27 & 0.37 & 0.47 & 0.47 & 0.25 \\
\hline & Ti-Lj & 0.35 & 0.44 & 0.30 & 0.21 & 0.28 & 0.37 & 0.37 & 0.20 \\
\hline & STi-Tj & 0.81 & 1.01 & 0.69 & 0.49 & 0.66 & 0.85 & 0.85 & 0.46 \\
\hline & SiL-jL & 0.91 & 1.13 & 0.77 & 0.55 & 0.73 & 0.95 & 0.95 & 0.51 \\
\hline & Sij-kl & 0.93 & 1.16 & 0.79 & 0.56 & 0.75 & 0.97 & 0.97 & 0.52 \\
\hline
\end{tabular}

*** Significantly deviating from zero for $5 \%$ and $1 \%$ level of significance 
Table.6 GCA and SCA effects for Cob girth $(\mathrm{cm})$ and 100-grain weight $(\mathrm{g})$

\begin{tabular}{|c|c|c|c|c|c|c|c|c|c|}
\hline \multirow[t]{2}{*}{ SN } & \multirow[t]{2}{*}{ Genotype } & \multicolumn{4}{|c|}{ Cob girth $(\mathrm{cm})$} & \multicolumn{4}{|c|}{ 100-grain weight (g): } \\
\hline & & E1 & E2 & E3 & Pool & E1 & E2 & E3 & Pool \\
\hline 1 & $\mathrm{~T}_{1}$ & $-0.16^{* *}$ & $-0.18^{* *}$ & $-0.17 * *$ & $-0.17 * *$ & $-2.44 * *$ & $-2.99 * *$ & $-2.01 * *$ & $-2.48 * *$ \\
\hline 2 & $\mathrm{~T}_{2}$ & -0.01 & 0.04 & $0.14 *$ & 0.06 & $0.90^{*}$ & $1.28 * *$ & $1.06 * *$ & $1.08^{* *}$ \\
\hline 3 & $\mathrm{~T}_{3}$ & $0.17 * *$ & $0.15^{* *}$ & 0.03 & $0.12 * *$ & $1.54 * *$ & $1.71 * *$ & $0.95^{*}$ & $1.40 * *$ \\
\hline 4 & $\mathrm{~L}_{1}$ & -0.18 & $-0.32 * *$ & $-0.46^{* *}$ & $-0.32 * *$ & $-2.75^{* *}$ & $-3.05^{* *}$ & $1.83^{*}$ & $-1.32 * *$ \\
\hline 5 & $\mathrm{~L}_{2}$ & $0.24 *$ & $0.38^{* *}$ & 0.22 & $0.28^{* * *}$ & $2.00^{* * *}$ & $2.22 *$ & $2.04 *$ & $2.09 * *$ \\
\hline 6 & $\mathrm{~L}_{3}$ & $-0.40 * *$ & $-0.43 * *$ & $-0.40 * *$ & $-0.41 * *$ & $-3.97 * *$ & $-5.82 * *$ & $-6.76^{* *}$ & $-5.52 * *$ \\
\hline 7 & $\mathrm{~L}_{4}$ & $-0.31 * *$ & $-0.28 * *$ & -0.22 & $-0.27 * *$ & $-3.04 * *$ & $-3.81 * *$ & $-5.38 * *$ & $-4.08 * *$ \\
\hline 8 & $\mathrm{~L}_{5}$ & 0.05 & -0.03 & 0.14 & 0.06 & 0.74 & 1.25 & 0.65 & 0.88 \\
\hline 9 & $\mathrm{~L}_{6}$ & $0.20^{*}$ & 0.01 & 0.10 & 0.10 & -0.15 & -0.00 & -0.60 & -0.25 \\
\hline 10 & $\mathrm{~L}_{7}$ & 0.12 & 0.13 & 0.22 & $0.16^{*}$ & -0.18 & 0.99 & 0.53 & 0.45 \\
\hline 11 & $\mathrm{~L}_{8}$ & 0.08 & 0.16 & 0.13 & $0.12 *$ & 1.07 & 1.27 & 0.67 & $1.00 *$ \\
\hline 12 & $\mathrm{~L}_{9}$ & $0.22^{*}$ & $0.33 * *$ & $0.55^{* *}$ & $0.37 * *$ & $-2.27 * *$ & $-1.98 *$ & -0.34 & $-1.53 * *$ \\
\hline 13 & $\mathrm{~L}_{10}$ & 0.15 & 0.17 & -0.14 & 0.06 & 0.22 & 0.62 & -0.12 & 0.24 \\
\hline 14 & $\mathrm{~L}_{11}$ & $-0.44 * *$ & $-0.47 * *$ & $-0.31 *$ & $-0.41 * *$ & $3.21 * *$ & $3.61 * *$ & $3.94 * *$ & $3.59 * *$ \\
\hline 15 & $\mathrm{~L}_{12}$ & $0.57 * *$ & $0.65^{* *}$ & $0.42 * *$ & $0.55^{* *}$ & $4.53 * *$ & $4.28 * *$ & $4.38 * *$ & $4.39 * *$ \\
\hline 16 & $\mathrm{~L}_{13}$ & 0.04 & -0.06 & 0.03 & 0.00 & 1.15 & $2.17 *$ & 0.91 & $1.41^{* *}$ \\
\hline 17 & $\mathrm{~L}_{14}$ & -0.16 & -0.10 & $-0.27 *$ & $-0.18^{* *}$ & $1.52 *$ & 0.05 & 0.94 & 0.84 \\
\hline 18 & $\mathrm{~L}_{15}$ & -0.19 & -0.14 & -0.04 & -0.12 & $-2.08^{* *}$ & $-1.79 *$ & $-2.68 * *$ & $-2.18 * *$ \\
\hline 19 & $\mathrm{~L}_{1} \times \mathrm{T}_{1}$ & 0.08 & 0.22 & -0.19 & 0.04 & $-8.77 * *$ & $-10.79 * *$ & 0.98 & $-6.19 * *$ \\
\hline 20 & $\mathrm{~L}_{2} \times \mathrm{T}_{1}$ & $-0.40 *$ & -0.12 & -0.11 & -0.21 & -0.74 & -0.36 & -1.76 & -0.95 \\
\hline 21 & $\mathrm{~L}_{3} \times \mathrm{T}_{1}$ & $0.50 *$ & -0.08 & 0.04 & 0.15 & 0.89 & -1.28 & -1.92 & -0.77 \\
\hline 22 & $\mathrm{~L}_{4} \times \mathrm{T}_{1}$ & 0.18 & 0.09 & -0.04 & 0.08 & 1.57 & 1.85 & 1.85 & 1.76 \\
\hline 23 & $\mathrm{~L}_{5} \times \mathrm{T}_{1}$ & $0.40 *$ & 0.30 & 0.03 & 0.24 & -0.13 & -1.51 & -2.48 & -1.37 \\
\hline 24 & $\mathrm{~L}_{6} \times \mathrm{T}_{1}$ & 0.12 & 0.23 & 0.36 & 0.24 & 0.47 & 0.91 & -0.06 & 0.44 \\
\hline 25 & $\mathrm{~L}_{7} \times \mathrm{T}_{1}$ & 0.07 & -0.25 & -0.24 & -0.14 & -1.25 & -2.06 & $-3.17 *$ & $-2.16^{*}$ \\
\hline 26 & $\mathrm{~L}_{8} \times \mathrm{T}_{1}$ & -0.31 & -0.19 & -0.23 & -0.24 & 1.80 & 2.33 & 1.35 & $1.83^{*}$ \\
\hline 27 & $\mathrm{~L}_{9} \times \mathrm{T}_{1}$ & $0.57 * *$ & $0.52^{*}$ & 0.42 & $0.50^{* *}$ & -0.06 & 0.38 & 1.42 & 0.58 \\
\hline 28 & $\mathrm{~L}_{10} \times \mathrm{T}_{1}$ & -0.10 & -0.04 & 0.29 & 0.05 & 0.02 & 0.34 & -0.22 & 0.05 \\
\hline 29 & $\mathrm{~L}_{11} \times \mathrm{T}_{1}$ & -0.15 & -0.20 & -0.21 & -0.19 & $3.95^{* *}$ & $4.64 * *$ & $3.25^{*}$ & $3.95^{* *}$ \\
\hline 30 & $\mathrm{~L}_{12} \times \mathrm{T}_{1}$ & -0.06 & 0.29 & $0.53 *$ & $0.25^{*}$ & 2.94 & $3.91 *$ & 2.23 & $3.02 * *$ \\
\hline 31 & $\mathrm{~L}_{13} \times \mathrm{T}_{1}$ & -0.03 & 0.05 & 0.07 & 0.03 & 0.69 & 0.40 & 0.08 & 0.39 \\
\hline 32 & $\mathrm{~L}_{14} \times \mathrm{T}_{1}$ & -0.35 & -0.34 & -0.18 & $-0.29 *$ & $-3.02 *$ & -0.83 & -2.96 & $-2.27 *$ \\
\hline 33 & $\mathrm{~L}_{15} \times \mathrm{T}_{1}$ & $-0.50 *$ & $-0.48^{*}$ & $-0.56^{*}$ & $-0.51^{* *}$ & 1.64 & 2.08 & 1.39 & 1.70 \\
\hline 34 & $\mathrm{~L}_{1} \times \mathrm{T}_{2}$ & -0.20 & -0.06 & 0.19 & -0.02 & $5.21 * *$ & $5.29 * *$ & 0.04 & $3.51 * *$ \\
\hline 35 & $\mathrm{~L}_{2} \times \mathrm{T}_{2}$ & $0.69^{* *}$ & $0.47 *$ & -0.09 & $0.36 * *$ & 1.91 & 3.01 & 2.81 & $2.57 * *$ \\
\hline 36 & $\mathrm{~L}_{3} \times \mathrm{T}_{2}$ & 0.01 & $0.42 *$ & 0.21 & 0.22 & -1.72 & -0.07 & 0.48 & -0.44 \\
\hline
\end{tabular}




\begin{tabular}{|c|c|c|c|c|c|c|c|c|c|}
\hline 37 & $\mathrm{~L}_{4} \times \mathrm{T}_{2}$ & -0.07 & -0.03 & -0.03 & -0.04 & 1.06 & 1.63 & 2.82 & $1.84 *$ \\
\hline 38 & $\mathrm{~L}_{5} \times \mathrm{T}_{2}$ & $-0.70 * *$ & $-0.60 * *$ & $-0.52 *$ & $-0.61 * *$ & 1.41 & 1.10 & 1.32 & 1.28 \\
\hline 39 & $\mathrm{~L}_{6} \times \mathrm{T}_{2}$ & -0.21 & -0.34 & -0.47 & $-0.34 * *$ & -2.36 & -2.71 & -2.49 & $-2.52 * *$ \\
\hline 40 & $\mathrm{~L}_{7} \times \mathrm{T}_{2}$ & $0.44 *$ & $0.89 * *$ & $0.65^{* *}$ & $0.66^{* *}$ & $3.79 *$ & $5.70 * *$ & $5.78 * *$ & $5.09 * *$ \\
\hline 41 & $\mathrm{~L}_{8} \times \mathrm{T}_{2}$ & 0.32 & 0.21 & 0.31 & $0.28 *$ & 0.03 & -0.36 & -0.14 & -0.16 \\
\hline 42 & $\mathrm{~L}_{9} \times \mathrm{T}_{2}$ & -0.18 & $-0.51^{*}$ & -0.29 & $-0.33^{*}$ & 0.80 & 0.97 & -0.73 & 0.35 \\
\hline 43 & $\mathrm{~L}_{10} \times \mathrm{T}_{2}$ & $-0.59 * *$ & $-0.71 * *$ & -0.35 & $-0.55 * *$ & -1.12 & -1.78 & -1.79 & -1.57 \\
\hline 44 & $\mathrm{~L}_{11} \times \mathrm{T}_{2}$ & -0.19 & -0.16 & -0.01 & -0.12 & -1.27 & -2.35 & -0.18 & -1.27 \\
\hline 45 & $\mathrm{~L}_{12} \times \mathrm{T}_{2}$ & -0.28 & $-0.50 *$ & -0.26 & $-0.34 * *$ & $-3.16^{*}$ & -2.25 & -2.18 & $-2.53^{* *}$ \\
\hline 46 & $\mathrm{~L}_{13} \times \mathrm{T}_{2}$ & 0.12 & 0.12 & -0.31 & -0.02 & 0.07 & -0.53 & -0.08 & -0.18 \\
\hline 47 & $\mathrm{~L}_{14} \times \mathrm{T}_{2}$ & 0.33 & 0.30 & 0.39 & $0.34 * *$ & 0.04 & -2.48 & -0.98 & -1.14 \\
\hline 48 & $\mathrm{~L}_{15} \times \mathrm{T}_{2}$ & $0.52 * *$ & $0.47 *$ & $0.57 *$ & $0.52 * *$ & $-4.67 * *$ & $-5.17 * *$ & $-4.67 * *$ & $-4.84 * *$ \\
\hline 49 & $\mathrm{~L}_{1} \times \mathrm{T}_{3}$ & 0.12 & -0.17 & -0.00 & -0.02 & $3.57^{*}$ & $5.50 * *$ & -1.02 & $2.68 * *$ \\
\hline 50 & $\mathrm{~L}_{2} \times \mathrm{T}_{3}$ & -0.29 & -0.36 & 0.20 & -0.15 & -1.17 & -2.64 & -1.05 & -1.62 \\
\hline 51 & $\mathrm{~L}_{3} \times \mathrm{T}_{3}$ & $-0.51 *$ & -0.34 & -0.25 & $-0.37 * *$ & 0.83 & 1.35 & 1.44 & 1.21 \\
\hline 52 & $\mathrm{~L}_{4} \times \mathrm{T}_{3}$ & -0.11 & -0.06 & 0.07 & -0.03 & -2.63 & $-3.49 *$ & $-4.67 * *$ & $-3.60 * *$ \\
\hline 53 & $\mathrm{~L}_{5} \times \mathrm{T}_{3}$ & 0.30 & 0.31 & $0.49 *$ & $0.36^{* *}$ & -1.28 & 0.41 & 1.16 & 0.10 \\
\hline 54 & $\mathrm{~L}_{6} \times \mathrm{T}_{3}$ & 0.09 & 0.11 & 0.11 & 0.10 & 1.88 & 1.79 & 2.55 & $2.07 *$ \\
\hline 55 & $\mathrm{~L}_{7} \times \mathrm{T}_{3}$ & $-0.51 *$ & $-0.64 * *$ & -0.41 & $-0.52 * *$ & -2.53 & $-3.65^{*}$ & -2.61 & $-2.93 * *$ \\
\hline 56 & $\mathrm{~L}_{8} \times \mathrm{T}_{3}$ & -0.01 & -0.01 & -0.08 & -0.03 & -1.83 & -1.97 & -1.21 & -1.67 \\
\hline 57 & $\mathrm{~L}_{9} \times \mathrm{T}_{3}$ & $-0.39 *$ & -0.02 & -0.13 & -0.18 & -0.75 & -1.35 & -0.69 & -0.93 \\
\hline 58 & $\mathrm{~L}_{10} \times \mathrm{T}_{3}$ & $0.69 * *$ & $0.75^{* *}$ & 0.05 & $0.50 * *$ & 1.10 & 1.44 & 2.01 & 1.52 \\
\hline 59 & $\mathrm{~L}_{11} \times \mathrm{T}_{3}$ & 0.34 & 0.36 & 0.22 & $0.31 *$ & -2.68 & -2.28 & -3.07 & $-2.68 * *$ \\
\hline 60 & $\mathrm{~L}_{12} \times \mathrm{T}_{3}$ & 0.34 & 0.21 & -0.27 & 0.09 & 0.23 & -1.66 & -0.05 & -0.49 \\
\hline 61 & $\mathrm{~L}_{13} \times \mathrm{T}_{3}$ & -0.09 & -0.17 & 0.24 & -0.01 & -0.75 & 0.13 & -0.00 & -0.21 \\
\hline 62 & $\mathrm{~L}_{14} \times \mathrm{T}_{3}$ & 0.03 & 0.04 & -0.21 & -0.05 & $2.98^{*}$ & 3.31 & $3.93^{*}$ & $3.41 * *$ \\
\hline \multirow[t]{11}{*}{63} & $\mathrm{~L}_{15} \times \mathrm{T}_{3}$ & -0.02 & 0.00 & -0.01 & -0.01 & $3.03 *$ & 3.10 & $3.28 *$ & $3.14 * *$ \\
\hline & \multicolumn{2}{|c|}{ Standard error } & & & & & & & \\
\hline & $\mathrm{Ti}$ & 0.05 & 0.05 & 0.06 & 0.03 & 0.38 & 0.43 & 0.40 & 0.23 \\
\hline & $\mathrm{Lj}$ & 0.10 & 0.11 & 0.12 & 0.06 & 0.75 & 0.85 & 0.79 & 0.46 \\
\hline & $\mathrm{Sij}$ & 0.19 & 0.21 & 0.25 & 0.13 & 1.50 & 1.71 & 1.58 & 0.92 \\
\hline & Ti-j & 0.06 & 0.07 & 0.08 & 0.04 & 0.46 & 0.52 & 0.48 & 0.28 \\
\hline & $\mathrm{Li}-\mathrm{j}$ & 0.13 & 0.15 & 0.17 & 0.09 & 1.03 & 1.17 & 1.08 & 0.63 \\
\hline & Ti-Lj & 0.10 & 0.11 & 0.13 & 0.07 & 0.80 & 0.91 & 0.84 & 0.49 \\
\hline & STi-Tj & 0.24 & 0.26 & 0.30 & 0.16 & 1.84 & 2.09 & 1.94 & 1.13 \\
\hline & SiL-jL & 0.27 & 0.29 & 0.34 & 0.17 & 2.06 & 2.34 & 2.16 & 1.26 \\
\hline & Sij-kl & 0.27 & 0.30 & 0.35 & 0.18 & 2.11 & 2.39 & 2.22 & 1.30 \\
\hline
\end{tabular}

*,** Significantly deviating from zero for $5 \%$ and $1 \%$ level of significance 
Table.7 GCA and SCA effects for Harvest index per cent and Grain Yield / Plant (g)

\begin{tabular}{|c|c|c|c|c|c|c|c|c|c|}
\hline \multirow[t]{2}{*}{ SN } & \multirow[t]{2}{*}{ Genotype } & \multicolumn{4}{|c|}{ Harvest index (\%) } & \multicolumn{4}{|c|}{ Grain Yield / Plant (g) } \\
\hline & & E1 & E2 & E3 & Pool & E1 & E2 & E3 & Pool \\
\hline 1 & $\mathrm{~T}_{1}$ & -0.12 & -0.46 & $1.58^{* *}$ & 0.33 & $12.10^{* *}$ & $3.21 *$ & 0.79 & $5.37 * *$ \\
\hline 2 & $\mathrm{~T}_{2}$ & -0.42 & -0.55 & $-1.09 *$ & $-0.68 * *$ & $5.12 * *$ & $5.54 * *$ & $4.13 * *$ & $4.93 * *$ \\
\hline 3 & $\mathrm{~T}_{3}$ & 0.54 & $1.01 * *$ & -0.49 & 0.35 & $-17.21 * *$ & $-8.75^{* *}$ & $-4.92 * *$ & $-10.30 * *$ \\
\hline 4 & $\mathrm{~L}_{1}$ & $-4.09 * *$ & $-1.71^{*}$ & -0.73 & $-2.18^{* *}$ & $8.77 * *$ & $10.32^{* * *}$ & $10.32 * *$ & $9.80 * *$ \\
\hline 5 & $\mathrm{~L}_{2}$ & -0.33 & $2.30^{* * *}$ & $2.08^{*}$ & $1.35^{*}$ & $5.82 *$ & $-5.73 *$ & -1.45 & -0.45 \\
\hline 6 & $\mathrm{~L}_{3}$ & 1.68 & -0.69 & -1.76 & -0.26 & $-23.94 * *$ & $-16.64 * *$ & $-10.77 * *$ & $-17.12 * *$ \\
\hline 7 & $\mathrm{~L}_{4}$ & 0.34 & $-2.68 * *$ & 0.29 & -0.68 & $-16.62 * *$ & -5.09 & $-6.55^{*}$ & $-9.42 * *$ \\
\hline 8 & $\mathrm{~L}_{5}$ & $2.16^{*}$ & $1.77^{*}$ & -0.53 & $1.13^{*}$ & $51.45^{* *}$ & $32.23 * *$ & $30.42 * *$ & $38.03 * *$ \\
\hline 9 & $\mathrm{~L}_{6}$ & 1.99 & 0.76 & -0.39 & 0.79 & $-12.78^{* *}$ & $-14.61 * *$ & $-17.26^{* *}$ & $-14.88^{* * *}$ \\
\hline 10 & $\mathrm{~L}_{7}$ & 0.52 & $1.89^{*}$ & 0.29 & 0.90 & $-8.74 * *$ & $-8.82 * *$ & $-17.04 * *$ & $-11.53 * *$ \\
\hline 11 & $\mathrm{~L}_{8}$ & 1.98 & -1.18 & -0.45 & 0.12 & $-11.62 * *$ & -4.20 & $-10.53 * *$ & $-8.79 * *$ \\
\hline 12 & $\mathrm{~L}_{9}$ & -1.05 & 1.16 & $3.05^{* *}$ & $1.05^{*}$ & -2.42 & -2.61 & $-9.71 * *$ & $-4.91 * *$ \\
\hline 13 & $\mathrm{~L}_{10}$ & 1.28 & -0.81 & $-1.97 *$ & -0.50 & $7.41 * *$ & 1.67 & $9.66^{* *}$ & $6.25 * *$ \\
\hline 14 & $\mathrm{~L}_{11}$ & 0.01 & $-2.10^{* *}$ & 1.66 & -0.14 & $-8.42 * *$ & $-5.62 *$ & -4.51 & $-6.18 * *$ \\
\hline 15 & $\mathrm{~L}_{12}$ & -0.12 & $1.53^{*}$ & -0.50 & 0.31 & $33.39 * *$ & $26.95^{* *}$ & $25.41 * *$ & $28.58 * *$ \\
\hline 16 & $\mathrm{~L}_{13}$ & $-3.00 * *$ & -0.83 & 0.63 & $-1.07 *$ & $-10.24 * *$ & -2.15 & 4.16 & -2.75 \\
\hline 17 & $\mathrm{~L}_{14}$ & -1.84 & $2.04 * *$ & -1.69 & -0.50 & $5.75^{*}$ & $12.02 * *$ & $13.61 * *$ & $10.46^{* *}$ \\
\hline 18 & $\mathrm{~L}_{15}$ & 0.46 & -1.47 & 0.03 & -0.33 & $-17.81 * *$ & $-17.73 * *$ & $-15.74 * *$ & $-17.09 * *$ \\
\hline 19 & $\mathrm{~L}_{1} \times \mathrm{T}_{1}$ & 3.76 & -2.33 & -0.12 & 0.44 & $-11.15^{*}$ & -4.04 & -9.09 & $-8.09 * *$ \\
\hline 20 & $\mathrm{~L}_{2} \times \mathrm{T}_{1}$ & 3.64 & -1.50 & -0.49 & 0.55 & $47.15^{* *}$ & $30.70 * *$ & $48.03 * *$ & $41.96 * *$ \\
\hline 21 & $\mathrm{~L}_{3} \times \mathrm{T}_{1}$ & 0.32 & $3.83^{*}$ & 1.02 & 1.72 & 8.56 & $14.58 * *$ & $17.66^{* *}$ & $13.60^{* *}$ \\
\hline 22 & $\mathrm{~L}_{4} \times \mathrm{T}_{1}$ & 0.30 & -0.53 & 3.28 & 1.02 & -0.63 & 10.17 & $15.58^{* *}$ & $8.37 * *$ \\
\hline 23 & $\mathrm{~L}_{5} \times \mathrm{T}_{1}$ & 0.39 & 2.82 & 1.23 & 1.48 & $-13.60 * *$ & 3.79 & -4.55 & -4.79 \\
\hline 24 & $\mathrm{~L}_{6} \times \mathrm{T}_{1}$ & -3.20 & 1.01 & 0.52 & -0.55 & -3.21 & $-14.12 *$ & $-16.09 * *$ & $-11.14 * *$ \\
\hline 25 & $\mathrm{~L}_{7} \times \mathrm{T}_{1}$ & -3.16 & -1.71 & -0.39 & -1.75 & $-37.96 * *$ & $-36.18 * *$ & $-40.01 * *$ & $-38.05^{* *}$ \\
\hline 26 & $\mathrm{~L}_{8} \times \mathrm{T}_{1}$ & 0.63 & 0.61 & -0.40 & 0.28 & $15.21 * *$ & -3.83 & -3.40 & 2.66 \\
\hline 27 & $\mathrm{~L}_{9} \times \mathrm{T}_{1}$ & 3.67 & -0.39 & -0.74 & 0.85 & $38.16^{* *}$ & $28.59 * *$ & 9.06 & $25.27 * *$ \\
\hline 28 & $\mathrm{~L}_{10} \times \mathrm{T}_{1}$ & 1.46 & 1.71 & 3.42 & $2.20^{*}$ & $-34.68 * *$ & $-26.16 * *$ & $-29.18^{* * *}$ & $-30.01 * *$ \\
\hline 29 & $\mathrm{~L}_{11} \times \mathrm{T}_{1}$ & -1.21 & -1.01 & 0.75 & -0.49 & 2.38 & 0.99 & 0.88 & 1.42 \\
\hline 30 & $\mathrm{~L}_{12} \times \mathrm{T}_{1}$ & 0.61 & $-3.95^{*}$ & -0.02 & -1.12 & -7.73 & 0.78 & -1.93 & -2.96 \\
\hline 31 & $\mathrm{~L}_{13} \times \mathrm{T}_{1}$ & -2.69 & -1.56 & -3.16 & $-2.47 *$ & -5.22 & -2.57 & $12.06^{*}$ & 1.42 \\
\hline 32 & $\mathrm{~L}_{14} \times \mathrm{T}_{1}$ & $-4.31 *$ & 3.04 & $-5.03 * *$ & $-2.10^{*}$ & -3.87 & 0.39 & -2.57 & -2.02 \\
\hline 33 & $\mathrm{~L}_{15} \times \mathrm{T}_{1}$ & -0.22 & -0.06 & 0.13 & -0.05 & 6.60 & -3.09 & 3.55 & 2.35 \\
\hline 34 & $\mathrm{~L}_{1} \times \mathrm{T}_{2}$ & -1.16 & -0.86 & 0.54 & -0.49 & $-22.88 * *$ & $-20.68 * *$ & -10.73 & $-18.09 * *$ \\
\hline 35 & $\mathrm{~L}_{2} \times \mathrm{T}_{2}$ & -3.50 & 0.32 & 0.40 & -0.93 & 5.41 & 5.46 & $-13.29 *$ & -0.81 \\
\hline 36 & $\mathrm{~L}_{3} \times \mathrm{T}_{2}$ & 0.34 & -0.61 & -1.95 & -0.74 & 0.49 & -4.40 & 1.35 & -0.85 \\
\hline
\end{tabular}




\begin{tabular}{|c|c|c|c|c|c|c|c|c|c|}
\hline 37 & $\mathrm{~L}_{4} \times \mathrm{T}_{2}$ & -0.05 & -0.90 & -2.07 & -1.01 & 8.51 & $11.06^{*}$ & -0.85 & $6.24 *$ \\
\hline 38 & $\mathrm{~L}_{5} \times \mathrm{T}_{2}$ & 0.39 & -0.75 & $-6.45^{* *}$ & $-2.27 *$ & $12.93 * *$ & -9.70 & -9.70 & -2.16 \\
\hline 39 & $\mathrm{~L}_{6} \times \mathrm{T}_{2}$ & $4.56^{*}$ & -2.19 & 0.16 & 0.84 & 6.97 & $11.21 *$ & $12.15^{*}$ & $10.11 * *$ \\
\hline 40 & $\mathrm{~L}_{7} \times \mathrm{T}_{2}$ & 2.30 & 2.28 & 0.69 & 1.76 & $39.39 * *$ & $32.03^{* * *}$ & $40.93 * *$ & $37.45^{* *}$ \\
\hline 41 & $\mathrm{~L}_{8} \times \mathrm{T}_{2}$ & 0.21 & 0.76 & 0.52 & 0.50 & -2.63 & $25.66^{* *}$ & $13.19^{*}$ & $12.07 * *$ \\
\hline 42 & $\mathrm{~L}_{9} \times \mathrm{T}_{2}$ & $-5.72 * *$ & -2.38 & 1.75 & $-2.12 *$ & $-35.13 * *$ & $-23.04 * *$ & $-15.65^{* *}$ & $-24.61 * *$ \\
\hline 43 & $\mathrm{~L}_{10} \times \mathrm{T}_{2}$ & -1.02 & -0.90 & 2.78 & 0.29 & -4.03 & -8.91 & -5.28 & -6.07 \\
\hline 44 & $\mathrm{~L}_{11} \times \mathrm{T}_{2}$ & 1.23 & 1.36 & 0.66 & 1.08 & $-24.42 * *$ & $-17.50 * *$ & -10.32 & $-17.42 * *$ \\
\hline 45 & $\mathrm{~L}_{12} \times \mathrm{T}_{2}$ & $5.74 * *$ & $3.16^{*}$ & -0.17 & $2.91 * *$ & $21.88 * *$ & -9.14 & -1.54 & 3.73 \\
\hline 46 & $\mathrm{~L}_{13} \times \mathrm{T}_{2}$ & -2.60 & 1.34 & $3.88^{*}$ & 0.87 & 5.50 & 10.46 & 5.76 & $7.24 *$ \\
\hline 47 & $\mathrm{~L}_{14} \times \mathrm{T}_{2}$ & -0.36 & -1.38 & -1.12 & -0.95 & 4.87 & 9.07 & 8.91 & $7.62 *$ \\
\hline 48 & $\mathrm{~L}_{15} \times \mathrm{T}_{2}$ & -0.37 & 0.74 & 0.36 & 0.24 & $-16.87 * *$ & $-11.57 *$ & $-14.93 *$ & $-14.46^{* *}$ \\
\hline 49 & $\mathrm{~L}_{1} \times \mathrm{T}_{3}$ & -2.60 & $3.18^{*}$ & -0.42 & 0.05 & $34.02 * *$ & $24.72 * *$ & $19.82^{* *}$ & $26.19 * *$ \\
\hline 50 & $\mathrm{~L}_{2} \times \mathrm{T}_{3}$ & -0.14 & 1.18 & 0.09 & 0.38 & $-52.56 * *$ & $-36.16 * *$ & $-34.74 * *$ & $-41.15^{* *}$ \\
\hline 51 & $\mathrm{~L}_{3} \times \mathrm{T}_{3}$ & -0.66 & $-3.23 *$ & 0.93 & -0.99 & -9.05 & -10.18 & $-19.01 * *$ & $-12.75^{* *}$ \\
\hline 52 & $\mathrm{~L}_{4} \times \mathrm{T}_{3}$ & -0.25 & 1.43 & -1.21 & -0.01 & -7.87 & $-21.23 * *$ & $-14.73 *$ & $-14.61 * *$ \\
\hline 53 & $\mathrm{~L}_{5} \times \mathrm{T}_{3}$ & -0.78 & -2.06 & $5.21 * *$ & 0.79 & 0.66 & 5.91 & $14.25^{*}$ & $6.94 *$ \\
\hline 54 & $\mathrm{~L}_{6} \times \mathrm{T}_{3}$ & -1.36 & 1.18 & -0.68 & -0.29 & -3.76 & 2.90 & 3.93 & 1.03 \\
\hline 55 & $\mathrm{~L}_{7} \times \mathrm{T}_{3}$ & 0.85 & -0.58 & -0.30 & -0.01 & -1.43 & 4.15 & -0.92 & 0.60 \\
\hline 56 & $\mathrm{~L}_{8} \times \mathrm{T}_{3}$ & -0.84 & -1.38 & -0.12 & -0.78 & $-12.58 *$ & $-21.83 * *$ & -9.79 & $-14.73^{* *}$ \\
\hline 57 & $\mathrm{~L}_{9} \times \mathrm{T}_{3}$ & 2.05 & 2.77 & -1.01 & 1.27 & -3.03 & -5.55 & 6.59 & -0.66 \\
\hline 58 & $\mathrm{~L}_{10} \times \mathrm{T}_{3}$ & -0.44 & -0.80 & $-6.20 * *$ & $-2.48 *$ & $38.72 * *$ & $35.07 * *$ & $34.46^{* *}$ & $36.08 * *$ \\
\hline 59 & $\mathrm{~L}_{11} \times \mathrm{T}_{3}$ & -0.02 & -0.36 & -1.41 & -0.59 & $22.03 * *$ & $16.52^{* * *}$ & 9.45 & $16.00 * *$ \\
\hline 60 & $\mathrm{~L}_{12} \times \mathrm{T}_{3}$ & $-6.35^{* *}$ & 0.79 & 0.19 & -1.79 & $-14.15^{* *}$ & 8.36 & 3.47 & -0.77 \\
\hline 61 & $\mathrm{~L}_{13} \times \mathrm{T}_{3}$ & $5.29 *$ & 0.22 & -0.72 & 1.60 & -0.27 & -7.89 & $-17.83 * *$ & $-8.66^{* *}$ \\
\hline 62 & $\mathrm{~L}_{14} \times \mathrm{T}_{3}$ & $4.67^{*}$ & -1.67 & $6.16^{* *}$ & $3.05 * *$ & -1.00 & -9.46 & -6.34 & -5.60 \\
\hline \multirow[t]{11}{*}{63} & $\mathrm{~L}_{15} \times \mathrm{T}_{3}$ & 0.59 & -0.68 & -0.50 & -0.20 & $10.27 *$ & $14.66^{* * *}$ & $11.39^{*}$ & $12.11^{* *}$ \\
\hline & \multicolumn{2}{|c|}{ Standard error } & & & & & & & \\
\hline & $\mathrm{Ti}$ & 0.51 & 0.38 & 0.45 & 0.26 & 1.22 & 1.37 & 1.44 & 0.78 \\
\hline & $\mathrm{Lj}$ & 1.03 & 0.77 & 0.91 & 0.52 & 2.44 & 2.73 & 2.88 & 1.55 \\
\hline & $\mathrm{Sij}$ & 2.05 & 1.54 & 1.82 & 1.05 & 4.88 & 5.47 & 5.76 & 3.11 \\
\hline & Ti-j & 0.63 & 0.47 & 0.56 & 0.32 & 1.49 & 1.67 & 1.76 & 0.95 \\
\hline & $\mathrm{Li}-\mathrm{j}$ & 1.40 & 1.05 & 1.24 & 0.72 & 3.34 & 3.74 & 3.94 & 2.13 \\
\hline & Ti-Lj & 1.09 & 0.82 & 0.96 & 0.56 & 2.59 & 2.90 & 3.05 & 1.65 \\
\hline & STi-Tj & 2.51 & 1.88 & 2.22 & 1.28 & 5.98 & 6.70 & 7.05 & 3.80 \\
\hline & SiL-jL & 2.81 & 2.11 & 2.49 & 1.43 & 6.68 & 7.49 & 7.88 & 4.25 \\
\hline & Sij-kl & 2.88 & 2.16 & 2.55 & 1.47 & 6.85 & 7.67 & 8.08 & 4.36 \\
\hline
\end{tabular}

*,** Significantly deviating from zero for $5 \%$ and $1 \%$ level of significance 
Table.8 GCA and SCA effects for Grain protein content in per cent, Grain starch content in per cent

\begin{tabular}{|c|c|c|c|c|c|c|c|c|c|}
\hline \multirow[t]{2}{*}{ SN } & \multirow[t]{2}{*}{ Genotype } & \multicolumn{4}{|c|}{ Grain protein content in (\%) } & \multicolumn{4}{|c|}{ Grain starch content in (\%) } \\
\hline & & E1 & E2 & E3 & Pool & E1 & E2 & E3 & Pool \\
\hline 1 & $\mathrm{~T}_{1}$ & $0.03 *$ & 0.02 & $-0.03 *$ & 0.01 & -0.05 & -0.10 & $0.15^{*}$ & -0.00 \\
\hline 2 & $T_{2}$ & $0.06 * *$ & 0.00 & $0.05^{* *}$ & $0.04 * *$ & $0.15^{*}$ & $0.20^{*}$ & 0.03 & $0.13^{* *}$ \\
\hline 3 & $\mathrm{~T}_{3}$ & $-0.09 * *$ & -0.02 & -0.02 & $.04 * *$ & -0.11 & -0.10 & $-0.18 *$ & $-0.13 * *$ \\
\hline 4 & $\mathrm{~L}_{1}$ & $-0.17 * *$ & $-0.23^{* *}$ & $-0.26^{* *}$ & $-0.22 * *$ & $1.88^{* * *}$ & $1.72 * *$ & $1.55^{* *}$ & $1.72 * *$ \\
\hline 5 & $\mathrm{~L}_{2}$ & $0.79 * *$ & $0.79 * *$ & $0.72 * *$ & $0.76^{* *}$ & $-1.71 * *$ & $-1.07 * *$ & $-0.68 * *$ & $-1.15^{* *}$ \\
\hline 6 & $\mathrm{~L}_{3}$ & $-0.37 * *$ & $-0.36^{* *}$ & $-0.50 * *$ & $-0.41 * *$ & $-1.30^{* *}$ & $-1.19 * *$ & $-1.15^{* *}$ & $-1.21 * *$ \\
\hline 7 & $\mathrm{~L}_{4}$ & $-0.33^{* *}$ & $-0.33^{* *}$ & $-0.41 * *$ & $-0.36^{* *}$ & $-0.36^{* *}$ & $-0.45^{*}$ & $-0.34 *$ & $-0.38 * *$ \\
\hline 8 & $\mathrm{~L}_{5}$ & -0.03 & $-0.15^{* *}$ & $0.26^{* *}$ & 0.03 & $-1.37 * *$ & $-1.32^{* *}$ & $-1.38^{* *}$ & $-1.36^{* *}$ \\
\hline 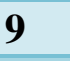 & $\mathrm{L}_{6}$ & $0.07 *$ & $0.09 * *$ & $0.10^{* * *}$ & $0.08 * *$ & $0.71 * *$ & $0.86^{* * *}$ & $1.55^{* *}$ & $1.04 * *$ \\
\hline 10 & $\mathrm{~L}_{7}$ & 0.04 & $0.42 * *$ & * & $0.27 * *$ & $-0.60 * *$ & $-0.70^{* *}$ & $-0.87 * *$ & $-0.72 * *$ \\
\hline 11 & $\mathrm{~L}_{8}$ & $0.25^{* *}$ & $0.09 * *$ & 0 . & 0.2 & $0.94 * *$ & $0.78 * *$ & -0.24 & $0.50^{* *}$ \\
\hline 12 & $\mathrm{~L}_{9}$ & $0.11 * *$ & 0.03 & 0.00 & $0.05 * *$ & $-1.44 * *$ & $-1.42 * *$ & $-1.58^{* *}$ & $-1.48 * *$ \\
\hline 13 & $\mathrm{~L}_{10}$ & $0.45^{* *}$ & $0.44 * *$ & $0.43 * *$ & $0.44 * *$ & $-1.36^{* *}$ & $-1.35^{* *}$ & $-0.59 * *$ & $-1.10 * *$ \\
\hline 14 & $\mathrm{~L}_{11}$ & $-0.30 * *$ & $-0.37 * *$ & -0.4 & -0.36 & $1.68 * *$ & $1.68 * *$ & .1 & $1.49 * *$ \\
\hline 15 & $\mathrm{~L}_{12}$ & $0.30 * *$ & $0.32 * *$ & & 0.25 & $3.13 * *$ & 2.7 & $2.25 * *$ & $2.70^{* *}$ \\
\hline 16 & $\mathrm{~L}_{13}$ & $-0.49 * *$ & $-0.47 * *$ & $-0.53 * *$ & $-0.50 * *$ & $-0.88^{* *}$ & $-0.68^{* *}$ & $-0.75^{* *}$ & $-0.77 * *$ \\
\hline 17 & $I_{H}$ & $-0.28 * *$ & $-0.28 * *$ & $-0.39 * *$ & $-0.32 * *$ & $0.48^{* * *}$ & 0.18 & $1.25^{* *}$ & $0.64^{* *}$ \\
\hline 18 & $\mathrm{~L}_{15}$ & -0.03 & 0.00 & -0.00 & -0.0 & 0.20 & 0.20 & -0.13 & 0.09 \\
\hline 19 & $\mathrm{~L}_{1} \mathrm{x}$ & $-0.29 * *$ & $-0.18 * *$ & $-0.14^{*}$ & $-0.20 * *$ & -0.33 & -0.09 & -0.12 & -0.18 \\
\hline 20 & $\mathrm{~L}_{2} \times \mathrm{T}_{1}$ & 0.02 & $0.11^{*}$ & $0.14^{*}$ & $0.09 * *$ & $2.02 * *$ & $1.46^{* *}$ & $0.87^{* *}$ & $1.45^{* *}$ \\
\hline 21 & $\mathrm{~L}_{3} \times \mathrm{T}_{1}$ & $0.46^{*}$ & $0.53 * *$ & $0.64 * *$ & $0.54 * *$ & $-3.16^{* *}$ & $-3.19 * *$ & $-3.44 * *$ & $-3.26^{* *}$ \\
\hline 22 & $\mathrm{~L}_{4} \times \mathrm{T}_{1}$ & 0.08 & 0.03 & & $0.07 *$ & $3.58^{* * *}$ & $3.75^{* * *}$ & $3.43^{* *}$ & $3.59 * *$ \\
\hline 23 & $\mathrm{~L}_{5} \times \mathrm{T}_{1}$ & $0.52 * *$ & $0.73 * *$ & $0.35^{* *}$ & $0.53 * *$ & $-0.88^{* *}$ & $-0.86^{*}$ & $-1.01 * *$ & $-0.92 * *$ \\
\hline 24 & $\mathrm{~L}_{6} \times \mathrm{T}_{1}$ & $0.38^{* *}$ & $0.43 * *$ & $0.54 * *$ & $0.45^{* *}$ & $-0.84^{* *}$ & $-0.91 *$ & $-1.80^{* *}$ & $-1.18^{* *}$ \\
\hline 25 & $\mathrm{~L}_{7} \times \mathrm{T}_{1}$ & $-0.44 * *$ & $-0.90 * *$ & $-0.73^{* *}$ & $-0.69 * *$ & $1.38 * *$ & $1.56^{* * *}$ & $1.53^{* *}$ & $1.49^{* *}$ \\
\hline 26 & $\mathrm{~L}_{8} \times \mathrm{T}_{1}$ & -0.08 & $-0.19 * *$ & & -0.26 & $-2.26^{* *}$ & $-2.02^{* *}$ & $-1.21 * *$ & $-1.83^{* *}$ \\
\hline 27 & $\mathrm{~L}_{9} \times \mathrm{T}_{1}$ & $0.35^{* *}$ & $0.42 * *$ & $0.44 * *$ & $0.40^{* *}$ & $3.31 * *$ & $3.37 * *$ & $2.79 * *$ & $3.16^{* *}$ \\
\hline 28 & $\mathrm{~L}_{10} \times \mathrm{T}_{1}$ & $-0.34 * *$ & $-0.39 * *$ & $-0.40 * *$ & $-0.38 * *$ & $-2.69 * *$ & $-2.62 * *$ & $-2.06^{* *}$ & $-2.46^{* *}$ \\
\hline 29 & $\mathrm{~L}_{11} \times \mathrm{T}_{1}$ & $-0.53^{* * *}$ & $-0.46 * *$ & $-0.45^{* *}$ & $-0.48 * *$ & $1.52^{* *}$ & $1.24 * *$ & $0.76^{* *}$ & $1.18 * *$ \\
\hline 30 & $\mathrm{~L}_{12} \times \mathrm{T}_{1}$ & $0.32 * *$ & $0.30 * *$ & & $0.35^{* *}$ & $2.81 * *$ & $2.94 * *$ & $3.27 * *$ & $3.01 * *$ \\
\hline 31 & $\mathrm{~L}_{13} \times \mathrm{T}_{1}$ & 0.03 & 0.06 & 0.10 & 0.06 & $-0.85^{* *}$ & $-1.12^{* *}$ & $-1.09 * *$ & $-1.02 * *$ \\
\hline 32 & $\mathrm{~L}_{14} \times \mathrm{T}_{1}$ & $0.17 * *$ & $0.16^{* *}$ & $0.17 * *$ & $0.17 * *$ & $-3.52^{* *}$ & $-3.48^{* *}$ & $-1.86^{* * *}$ & $-2.95^{* *}$ \\
\hline 33 & $\mathrm{~L}_{15} \times \mathrm{T}_{1}$ & $-0.64 * *$ & $-0.65 * *$ & $-0.68 * *$ & $-0.65 * *$ & -0.09 & -0.01 & -0.05 & -0.05 \\
\hline 34 & $\mathrm{~L}_{1} \times \mathrm{T}_{2}$ & -0 . & -0.06 & & -0 . & $-1.37^{* * *}$ & $-1.24 * *$ & -0.44 & $-1.02 * *$ \\
\hline 35 & $\mathrm{~L}_{2} \times \mathrm{T}_{2}$ & $0.12 *$ & $0.12^{*}$ & -0.03 & $0.07 *$ & 0.09 & -0.57 & $-0.75^{* *}$ & $-0.41 *$ \\
\hline
\end{tabular}




\begin{tabular}{|c|c|c|c|c|c|c|c|c|c|}
\hline 36 & $\mathrm{~L}_{3} \times \mathrm{T}_{2}$ & $-0.12 *$ & -0.05 & -0.03 & $-0.07 *$ & $-1.15^{* *}$ & $-1.28 * *$ & $-1.11 * *$ & $-1.18 * *$ \\
\hline 37 & $\mathrm{~L}_{4} \times \mathrm{T}_{2}$ & $0.19 * *$ & $0.23 * *$ & $0.16 * *$ & $0.19 * *$ & 0.03 & 0.09 & 0.20 & 0.11 \\
\hline 38 & $\mathrm{~L}_{5} \times \mathrm{T}_{2}$ & $-0.18^{* *}$ & $-0.39 * *$ & $-0.73^{* *}$ & $-0.44 * *$ & $4.52 * *$ & $4.44 * *$ & $4.49 * *$ & $4.49^{* *}$ \\
\hline 39 & $\mathrm{~L}_{6} \times \mathrm{T}_{2}$ & $-0.35^{* *}$ & $-0.29 * *$ & $-0.28 * *$ & $-0.31 * *$ & $-1.74 * *$ & $-1.92 * *$ & $-0.68^{*}$ & $-1.45 * *$ \\
\hline 40 & $\mathrm{~L}_{7} \times \mathrm{T}_{2}$ & $0.87 * *$ & $0.71 * *$ & $0.67 * *$ & $0.75^{* *}$ & $0.95 * *$ & $1.02 * *$ & 0.38 & $0.78^{* *}$ \\
\hline 41 & $\mathrm{~L}_{8} \times \mathrm{T}_{2}$ & $-0.29 * *$ & $-0.43^{* *}$ & $0.27 * *$ & $-0.15^{* *}$ & $2.18 * *$ & $2.31 * *$ & 0.04 & $1.51^{* *}$ \\
\hline 42 & $\mathrm{~L}_{9} \times \mathrm{T}_{2}$ & $-0.31 * *$ & $-0.29 * *$ & $-0.37 * *$ & $-0.32 * *$ & $-3.04 * *$ & $-3.08 * *$ & $-2.70 * *$ & $-2.94 * *$ \\
\hline 43 & $\mathrm{~L}_{10} \times \mathrm{T}_{2}$ & $0.16^{* *}$ & $0.22 * *$ & $0.18 * *$ & $0.19 * *$ & 0.34 & 0.30 & -0.39 & 0.09 \\
\hline 44 & $\mathrm{~L}_{11} \times \mathrm{T}_{2}$ & $0.22 * *$ & $0.26 * *$ & $0.34 * *$ & $0.27 * *$ & $-0.87 * *$ & -0.59 & -0.11 & $-0.52 * *$ \\
\hline 45 & $\mathrm{~L}_{12} \times \mathrm{T}_{2}$ & $-0.43 * *$ & $-0.45^{* * *}$ & $-0.48^{* *}$ & $-0.45 * *$ & $-3.16^{* *}$ & $-3.07 * *$ & $-1.99 * *$ & $-2.74 * *$ \\
\hline 46 & $\mathrm{~L}_{13} \times \mathrm{T}_{2}$ & -0.01 & 0.09 & 0.05 & 0.04 & 0.21 & -0.01 & 0.27 & 0.16 \\
\hline 47 & $\mathrm{~L}_{14} \times \mathrm{T}_{2}$ & $-0.40 * *$ & $-0.32 * *$ & $-0.37 * *$ & $-0.36^{* *}$ & $2.46^{* *}$ & $2.90 * *$ & $2.22 * *$ & $2.53^{* *}$ \\
\hline 48 & $\mathrm{~L}_{15} \times \mathrm{T}_{2}$ & $0.53^{* *}$ & $0.64 * *$ & $0.68^{* *}$ & $0.62 * *$ & $0.55^{*}$ & 0.70 & $0.57^{*}$ & $0.61^{* *}$ \\
\hline 49 & $\mathrm{~L}_{1} \times \mathrm{T}_{3}$ & $0.29 * *$ & $0.24 * *$ & $0.18 * *$ & $0.24 * *$ & $1.71 * *$ & $1.33 * *$ & $0.56^{*}$ & $1.20 * *$ \\
\hline 50 & $\mathrm{~L}_{2} \times \mathrm{T}_{3}$ & $-0.14^{*}$ & $-0.23 * *$ & -0.11 & $-0.16^{* *}$ & $-2.10 * *$ & $-0.88^{*}$ & -0.12 & $-1.03 * *$ \\
\hline 51 & $\mathrm{~L}_{3} \times \mathrm{T}_{3}$ & $-0.34 * *$ & $-0.48^{* *}$ & $-0.60 * *$ & $-0.48 * *$ & $4.32 * *$ & $4.47 * *$ & $4.55^{* *}$ & $4.44 * *$ \\
\hline 52 & $\mathrm{~L}_{4} \times \mathrm{T}_{3}$ & $-0.27 * *$ & $-0.26^{* *}$ & $-0.24 * *$ & $-0.26^{* *}$ & $-3.61^{* *}$ & $-3.84 * *$ & $-3.63^{* *}$ & $-3.69 * *$ \\
\hline 53 & $\mathrm{~L}_{5} \times \mathrm{T}_{3}$ & $-0.33 * *$ & $-0.34 * *$ & $0.38 * *$ & $-0.10 * *$ & $-3.64 * *$ & $-3.58 * *$ & $-3.48 * *$ & $-3.57 * *$ \\
\hline 54 & $\mathrm{~L}_{6} \times \mathrm{T}_{3}$ & -0.02 & $-0.14 * *$ & $-0.26^{* *}$ & $-0.14 * *$ & $2.58 * *$ & $2.83 * *$ & $2.48 * *$ & $2.63 * *$ \\
\hline 55 & $\mathrm{~L}_{7} \times \mathrm{T}_{3}$ & $-0.43 * *$ & $0.19^{* *}$ & 0.06 & -0.06 & $-2.32 * *$ & $-2.58 * *$ & $-1.91 * *$ & $-2.27 * *$ \\
\hline 56 & $\mathrm{~L}_{8} \times \mathrm{T}_{3}$ & $0.38 * *$ & $0.62 * *$ & $0.24 * *$ & $0.41 * *$ & 0.08 & -0.29 & $1.17 * *$ & 0.32 \\
\hline 57 & $\mathrm{~L}_{9} \times \mathrm{T}_{3}$ & -0.05 & $-0.13 *$ & -0.07 & $-0.08^{*}$ & -0.28 & -0.28 & -0.09 & -0.22 \\
\hline 58 & $\mathrm{~L}_{10} \times \mathrm{T}_{3}$ & $0.18 * *$ & $0.16^{* *}$ & $0.23 * *$ & $0.19 * *$ & $2.35 * *$ & $2.32 * *$ & $2.45 * *$ & $2.37 * *$ \\
\hline 59 & $\mathrm{~L}_{11} \times \mathrm{T}_{3}$ & $0.31 * *$ & $0.20 * *$ & 0.11 & $0.21 * *$ & $-0.66^{*}$ & -0.65 & $-0.65^{*}$ & $-0.65 * *$ \\
\hline 60 & $\mathrm{~L}_{12} \times \mathrm{T}_{3}$ & 0.11 & $0.15 * *$ & 0.05 & $0.10 * *$ & 0.34 & 0.13 & $-1.28 * *$ & -0.27 \\
\hline 61 & $\mathrm{~L}_{13} \times \mathrm{T}_{3}$ & -0.02 & $-0.15^{* *}$ & $-0.15^{*}$ & $-0.10^{* *}$ & $0.65^{*}$ & $1.13 * *$ & $0.82 * *$ & $0.87 * *$ \\
\hline 62 & $\mathrm{~L}_{14} \times \mathrm{T}_{3}$ & $0.23 * *$ & $0.16^{* *}$ & $0.19 * *$ & $0.19 * *$ & $1.06^{* *}$ & 0.58 & -0.36 & $0.43 *$ \\
\hline \multirow[t]{11}{*}{63} & $\mathrm{~L}_{15} \times \mathrm{T}_{3}$ & 0.11 & 0.00 & -0.00 & 0.04 & -0.47 & -0.69 & -0.52 & $-0.56^{* *}$ \\
\hline & \multicolumn{2}{|c|}{ Standard error } & & & & & & & \\
\hline & $\mathrm{Ti}$ & 0.01 & 0.01 & 0.01 & 0.01 & 0.06 & 0.10 & 0.07 & 0.05 \\
\hline & $\mathrm{Lj}$ & 0.03 & 0.03 & 0.03 & 0.02 & 0.13 & 0.19 & 0.14 & 0.09 \\
\hline & $\mathrm{Sij}$ & 0.06 & 0.05 & 0.06 & 0.03 & 0.26 & 0.38 & 0.28 & 0.18 \\
\hline & Ti-j & 0.02 & 0.02 & 0.02 & 0.01 & 0.08 & 0.12 & 0.09 & 0.06 \\
\hline & $\mathrm{Li}-\mathrm{j}$ & 0.04 & 0.04 & 0.04 & 0.02 & 0.18 & 0.26 & 0.19 & 0.12 \\
\hline & Ti-Lj & 0.03 & 0.03 & 0.03 & 0.02 & 0.14 & 0.20 & 0.15 & 0.10 \\
\hline & $\mathrm{STi}-\mathrm{Tj}$ & 0.07 & 0.06 & 0.07 & 0.04 & 0.31 & 0.47 & 0.34 & 0.22 \\
\hline & SiL-jL & 0.08 & 0.07 & 0.08 & 0.04 & 0.35 & 0.53 & 0.38 & 0.25 \\
\hline & Sij-kl & 0.08 & 0.07 & 0.08 & 0.05 & 0.36 & 0.54 & 0.39 & 0.25 \\
\hline
\end{tabular}

*** Significantly deviating from zero for $5 \%$ and $1 \%$ level of significance 
Table.9 GCA and SCA effects for Grain Oil content in per cent

\begin{tabular}{|c|c|c|c|c|c|}
\hline \multirow[t]{2}{*}{ SN } & \multirow[t]{2}{*}{ Genotype } & \multicolumn{4}{|c|}{ Grain Oil content in $(\%)$} \\
\hline & & E1 & E2 & E3 & Pool \\
\hline 1 & $\mathrm{~T}_{1}$ & $-0.11 * *$ & $-0.12 * *$ & $-0.02 * *$ & $-0.08 * *$ \\
\hline 2 & $\mathrm{~T}_{2}$ & $0.05^{* *}$ & $0.06 * *$ & $-0.03^{* *}$ & $0.03 * *$ \\
\hline 3 & $\mathrm{~T}_{3}$ & $0.06^{* *}$ & $0.06^{* *}$ & $0.05^{* *}$ & $0.06^{* *}$ \\
\hline 4 & $\mathrm{~L}_{1}$ & $0.16^{* *}$ & $0.18^{* *}$ & $0.09 * *$ & $0.14^{* *}$ \\
\hline 5 & $\mathrm{~L}_{2}$ & $0.14 * *$ & $0.15^{* *}$ & $0.12 * *$ & $0.14 * *$ \\
\hline 6 & $\mathrm{~L}_{3}$ & $0.20 * *$ & $0.20 * *$ & $0.21 * *$ & $0.20 * *$ \\
\hline 7 & $\mathrm{~L}_{4}$ & $-0.61 * *$ & $-0.61 * *$ & $-0.52 * *$ & $-0.58 * *$ \\
\hline 8 & $\mathrm{~L}_{5}$ & $0.16^{* *}$ & $0.17 * *$ & $0.13 * *$ & $0.15^{* *}$ \\
\hline 9 & $\mathrm{~L}_{6}$ & $0.12 * *$ & $0.11^{* *}$ & $0.24 * *$ & $0.16^{* * *}$ \\
\hline 10 & $\mathrm{~L}_{7}$ & -0.01 & $-0.04 *$ & $0.03 *$ & -0.01 \\
\hline 11 & $\mathrm{~L}_{8}$ & 0.02 & 0.01 & $-0.11 * *$ & $-0.03 * *$ \\
\hline 12 & $\mathrm{~L}_{9}$ & $0.12 * *$ & $0.11 * *$ & -0.01 & $0.07 * *$ \\
\hline 13 & $\mathrm{~L}_{10}$ & $0.05^{* *}$ & $0.04 * *$ & $-0.07 * *$ & 0.01 \\
\hline 14 & $\mathrm{~L}_{11}$ & $-0.40 * *$ & $-0.42 * *$ & $-0.39 * *$ & $-0.40 * *$ \\
\hline 15 & $\mathrm{~L}_{12}$ & $0.40^{* *}$ & $0.41 * *$ & $0.55^{* *}$ & $0.46^{* *}$ \\
\hline 16 & $\mathrm{~L}_{13}$ & $-0.11 * *$ & $-0.10 * *$ & $-0.13 * *$ & $-0.11 * *$ \\
\hline 17 & $\mathrm{~L}_{14}$ & $-0.10^{* *}$ & $-0.08 * *$ & $-0.04 * *$ & $-0.07 * *$ \\
\hline 18 & $\mathrm{~L}_{15}$ & $-0.14 * *$ & $-0.12 * *$ & $-0.09 * *$ & $-0.12 * *$ \\
\hline 19 & $\mathrm{~L}_{1} \times \mathrm{T}_{1}$ & $-0.33^{* *}$ & $-0.29 * *$ & $-0.30 * *$ & $-0.31 * *$ \\
\hline 20 & $\mathrm{~L}_{2} \times \mathrm{T}_{1}$ & $0.35^{* *}$ & $0.34 * *$ & $0.26^{* *}$ & $0.32 * *$ \\
\hline 21 & $\mathrm{~L}_{3} \times \mathrm{T}_{1}$ & $0.18^{* *}$ & $0.17 * *$ & $0.10^{* *}$ & $0.15^{* *}$ \\
\hline 22 & $\mathrm{~L}_{4} \times \mathrm{T}_{1}$ & 0.04 & 0.03 & 0.01 & 0.03 \\
\hline 23 & $\mathrm{~L}_{5} \times \mathrm{T}_{1}$ & $0.20 * *$ & $0.19 * *$ & 0.03 & $0.14^{* *}$ \\
\hline 24 & $\mathrm{~L}_{6} \times \mathrm{T}_{1}$ & $0.26 * *$ & $0.24 * *$ & $0.30^{* *}$ & $0.27 * *$ \\
\hline 25 & $\mathrm{~L}_{7} \times \mathrm{T}_{1}$ & $-0.21 * *$ & $-0.21 * *$ & $-0.29 * *$ & $-0.23 * *$ \\
\hline 26 & $\mathrm{~L}_{8} \times \mathrm{T}_{1}$ & $-0.11 * *$ & $-0.11 * *$ & $-0.15^{* *}$ & $-0.12 * *$ \\
\hline 27 & $\mathrm{~L}_{9} \times \mathrm{T}_{1}$ & $0.55^{* *}$ & $0.54 * *$ & $0.56^{* *}$ & $0.55^{* *}$ \\
\hline 28 & $\mathrm{~L}_{10} \times \mathrm{T}_{1}$ & $-0.33^{* *}$ & $-0.32 * *$ & $-0.33 * *$ & $-0.32 * *$ \\
\hline 29 & $\mathrm{~L}_{11} \times \mathrm{T}_{1}$ & 0.01 & -0.04 & $-0.06^{* *}$ & $-0.03 *$ \\
\hline 30 & $\mathrm{~L}_{12} \times \mathrm{T}_{1}$ & 0.01 & 0.02 & $0.18 * *$ & $0.07 * *$ \\
\hline 31 & $\mathrm{~L}_{13} \times \mathrm{T}_{1}$ & -0.06 & -0.04 & $0.14 * *$ & 0.01 \\
\hline 32 & $\mathrm{~L}_{14} \times \mathrm{T}_{1}$ & $-0.28 * *$ & $-0.26^{* *}$ & $-0.19 * *$ & $-0.24 * *$ \\
\hline 33 & $\mathrm{~L}_{15} \times \mathrm{T}_{1}$ & $-0.29 * *$ & $-0.27 * *$ & $-0.26 * *$ & $-0.27 * *$ \\
\hline 34 & $\mathrm{~L}_{1} \times \mathrm{T}_{2}$ & 0.06 & 0.04 & $-0.19 * *$ & -0.03 \\
\hline 35 & $\mathrm{~L}_{2} \times \mathrm{T}_{2}$ & $0.24 * *$ & $0.24 * *$ & $0.34 * *$ & $0.27 * *$ \\
\hline 36 & $\mathrm{~L}_{3} \times \mathrm{T}_{2}$ & $-0.34 * *$ & $-0.35^{* *}$ & $-0.27 * *$ & $-0.32^{* *}$ \\
\hline
\end{tabular}




\begin{tabular}{|c|c|c|c|c|c|}
\hline 37 & $\mathrm{~L}_{4} \times \mathrm{T}_{2}$ & $0.13^{* * *}$ & $0.13^{* *}$ & $0.08 * *$ & $0.11 * *$ \\
\hline 38 & $\mathrm{~L}_{5} \times \mathrm{T}_{2}$ & $-0.50^{* *}$ & $-0.51 * *$ & $-0.42 * *$ & $-0.48 * *$ \\
\hline 39 & $\mathrm{~L}_{6} \times \mathrm{T}_{2}$ & -0.02 & -0.02 & $-0.16^{* *}$ & $-0.07 * *$ \\
\hline 40 & $\mathrm{~L}_{7} \times \mathrm{T}_{2}$ & $0.21 * *$ & $0.19 * *$ & $0.33^{* *}$ & $0.24 * *$ \\
\hline 41 & $\mathrm{~L}_{8} \times \mathrm{T}_{2}$ & $-0.07^{*}$ & $-0.06^{*}$ & $-0.19 * *$ & $-0.11 * *$ \\
\hline 42 & $\mathrm{~L}_{9} \times \mathrm{T}_{2}$ & $-0.11^{* *}$ & $-0.11 * *$ & $-0.23 * *$ & $-0.15^{* *}$ \\
\hline 43 & $\mathrm{~L}_{10} \times \mathrm{T}_{2}$ & $-0.27 * *$ & $-0.29 * *$ & $-0.06^{* *}$ & $-0.21^{* *}$ \\
\hline 44 & $\mathrm{~L}_{11} \times \mathrm{T}_{2}$ & $-0.07^{*}$ & -0.02 & -0.04 & $-0.04 * *$ \\
\hline 45 & $\mathrm{~L}_{12} \times \mathrm{T}_{2}$ & $0.07^{*}$ & $0.08^{* *}$ & -0.00 & $0.05^{* *}$ \\
\hline 46 & $\mathrm{~L}_{13} \times \mathrm{T}_{2}$ & $0.09 * *$ & $0.09 * *$ & $0.21^{* *}$ & $0.13 * *$ \\
\hline 47 & $\mathrm{~L}_{14} \times \mathrm{T}_{2}$ & $0.45^{* *}$ & $0.45^{* *}$ & $0.48^{* * *}$ & $0.46^{* * *}$ \\
\hline 48 & $\mathrm{~L}_{15} \times \mathrm{T}_{2}$ & $0.13 * *$ & $0.15^{* *}$ & $0.14 * *$ & $0.14 * *$ \\
\hline 49 & $\mathrm{~L}_{1} \times \mathrm{T}_{3}$ & $0.27 * *$ & $0.26^{* *}$ & $0.49 * *$ & $0.34 * *$ \\
\hline 50 & $\mathrm{~L}_{2} \times \mathrm{T}_{3}$ & $-0.59 * *$ & $-0.58 * *$ & $-0.59 * *$ & $-0.59 * *$ \\
\hline 51 & $\mathrm{~L}_{3} \times \mathrm{T}_{3}$ & $0.17^{* *}$ & $0.18^{* *}$ & $0.17 * *$ & $0.17 * *$ \\
\hline 52 & $\mathrm{~L}_{4} \times \mathrm{T}_{3}$ & $-0.17 * *$ & $-0.16^{* *}$ & $-0.09 * *$ & $-0.14 * *$ \\
\hline 53 & $\mathrm{~L}_{5} \times \mathrm{T}_{3}$ & $0.29 * *$ & $0.32 * *$ & $0.39 * *$ & $0.33^{* *}$ \\
\hline 54 & $\mathrm{~L}_{6} \times \mathrm{T}_{3}$ & $-0.24 * *$ & $-0.22 * *$ & $-0.14 * *$ & $-0.20 * *$ \\
\hline 55 & $\mathrm{~L}_{7} \times \mathrm{T}_{3}$ & -0.00 & 0.02 & -0.04 & -0.01 \\
\hline 56 & $\mathrm{~L}_{8} \times \mathrm{T}_{3}$ & $0.18 * *$ & $0.18^{* *}$ & $0.34 * *$ & $0.23 * *$ \\
\hline 57 & $\mathrm{~L}_{9} \times \mathrm{T}_{3}$ & $-0.43^{* *}$ & $-0.43^{* *}$ & $-0.33^{* *}$ & $-0.40^{* *}$ \\
\hline 58 & $\mathrm{~L}_{10} \times \mathrm{T}_{3}$ & $0.60 * *$ & $0.60 * *$ & $0.39 * *$ & $0.53 * *$ \\
\hline 59 & $\mathrm{~L}_{11} \times \mathrm{T}_{3}$ & 0.06 & $0.07^{*}$ & $0.11^{* *}$ & $0.08^{* *}$ \\
\hline 60 & $\mathrm{~L}_{12} \times \mathrm{T}_{3}$ & $-0.08^{*}$ & $-0.10 * *$ & $-0.18 * *$ & $-0.12 * *$ \\
\hline 61 & $\mathrm{~L}_{13} \times \mathrm{T}_{3}$ & -0.03 & -0.05 & $-0.34 * *$ & $-0.14 * *$ \\
\hline 62 & $\mathrm{~L}_{14} \times \mathrm{T}_{3}$ & $-0.18^{* *}$ & $-0.20 * *$ & $-0.29 * *$ & $-0.22 * *$ \\
\hline \multirow[t]{11}{*}{63} & $\mathrm{~L}_{15} \times \mathrm{T}_{3}$ & $0.15^{* *}$ & $0.13^{* *}$ & $0.11^{* *}$ & $0.13^{* *}$ \\
\hline & Standard error & & & & \\
\hline & $\mathrm{Ti}$ & 0.01 & 0.01 & 0.01 & 0.00 \\
\hline & $\mathrm{Lj}$ & 0.02 & 0.01 & 0.01 & 0.01 \\
\hline & Sij & 0.03 & 0.03 & 0.02 & 0.02 \\
\hline & Ti-j & 0.01 & 0.01 & 0.01 & 0.01 \\
\hline & $\mathrm{Li}-\mathrm{j}$ & 0.02 & 0.02 & 0.02 & 0.01 \\
\hline & Ti-Lj & 0.02 & 0.02 & 0.01 & 0.01 \\
\hline & STi-Tj & 0.04 & 0.04 & 0.03 & 0.02 \\
\hline & SiL-jL & 0.04 & 0.04 & 0.03 & 0.02 \\
\hline & Sij-kl & 0.05 & 0.04 & 0.03 & 0.02 \\
\hline
\end{tabular}

*,** Significantly deviating from zero for $5 \%$ and $1 \%$ level of significance 
Analysis of variance for combining ability revealed (Table 1) that mean squares due to lines, testers and lines $\mathrm{x}$ tester were significant for all the characters in all the environments. Significant mean squares due to lines and testers for different characters indicated significant contribution of lines and testers towards the general combining (gca) effects. Similarly significant mean squares due to lines $\mathrm{x}$ testers for different characters indicated significant contribution of hybrids for specific combining (sca) effects.

Results on pooled basis (Table 2) revealed that mean squares due to lines, testers and lines $\mathrm{x}$ testers were significant for all the characters. The interaction of lines with environments was significant for all the characters except days to 50 per cent tasseling, days to 50 per cent silking, days to 75 per cent brown husk, ear height, number of grain rows/ear, cob girth. Further, testers $\mathrm{x}$ environments interaction was significant for all the characters except days to 50 per cent tasseling, days to 50 per cent silking, days to 75 per cent \% brown husk, plant height, ear height, cob girth, 100-grain weight. The interaction of lines $\mathrm{x}$ testers $\mathrm{x}$ environments were significant for all the characters except days to 50 per cent tasseling, days to 50 per cent silking, days to 75 per cent brown husk, ear height, number of grain rows/ear. This indicated that estimates of gca and sca effects were highly influenced by environments for large numbers of characters studied (Table 3).

Parents were classified as high or good, medium or average and low or poor combiners on the basis of their gca effects. Parents with desirable and gca effects were considered high or good combiners while parents showing non-significant estimates but in desirable direction were classified as average or medium combiners. Poor or low combiners had undesirable gca effects. The estimates of gca effects revealed that the good general combiner for yield were inbred lines $\mathrm{L}_{1}, \mathrm{~L}_{5}, \mathrm{~L}_{10}, \mathrm{~L}_{12}$, and $\mathrm{L}_{14}$, for oil content inbred lines $\mathrm{L}_{1}, \mathrm{~L}_{2}, \mathrm{~L}_{3}, \mathrm{~L}_{5}, \mathrm{~L}_{6}, \mathrm{~L}_{9}$, and $\mathrm{L}_{12}$, for protein content inbred lines $\mathrm{L}_{2}, \mathrm{~L}_{6}, \mathrm{~L}_{7}, \mathrm{~L}_{8}, \mathrm{~L}_{9}, \mathrm{~L}_{10}$, and $\mathrm{L}_{12}$, for starch content inbred lines $\mathrm{L}_{1}, \mathrm{~L}_{6}, \mathrm{~L}_{8}$, $\mathrm{L}_{11}, \mathrm{~L}_{12}$, and $\mathrm{L}_{14}$, for brown husk related traits inbred lines $\mathrm{L}_{4}, \mathrm{~L}_{5}, \mathrm{~L}_{10}$ and $\mathrm{L}_{12}$, for plant type traits inbred lines $\mathrm{L}_{3}, \mathrm{~L}_{5}$ and $\mathrm{L}_{10}$ (Table 3). Among the testers, $\mathrm{T}_{1}$ was considered good general combiner for brown husk related traits, protein content. The tester $\mathrm{T}_{2}$ was considered good general combiner for brown husk related traits, starch content. Tester $\mathrm{T}_{3}$ was considered good general combiner for yield and yield contributing traits and oil content. The general combining effects observed is due to additive gene effects and additive $\mathrm{x}$ additive gene effects (Griffing, 1956 and Sprague, 1966).

A perusal of sca effect revealed that highest magnitude of positive significant sca effects for grain yield per plant was recorded in hybrid $\mathrm{L}_{2} \times \mathrm{T}_{1}$ in $\mathrm{E}_{1}$ (47.15), in $\mathrm{E}_{2}$ (30.70), in $\mathrm{E}_{3}$ (48.03) and on pooled basis (41.96). Three hybrids viz., $\mathrm{L}_{7} \times \mathrm{T}_{2}, \mathrm{~L}_{1} \times \mathrm{T}_{3}$ and $\mathrm{L}_{10} \times \mathrm{T}_{3}$ exhibited positive significant sca effects in all the three environments as well as on pooled basis. Hybrid $\mathrm{L}_{10} \times \mathrm{T}_{3}$ besides grain yield per plant also exhibited positive significant sca effects for cob length, numbers of grain rows per ear, cob girth, starch content, oil content. Hybrid $L_{7} \times T_{2}$ in addition to grain yield per plant also exhibited significant positive sca effects for cob length, numbers of grain rows per ear, cob girth, 100-grain weight (Table 49).

Out of 45 hybrids five best hybrids which exhibited positive significant sca effects for grain yield per plant are viz., $\mathrm{L}_{2} \times \mathrm{T}_{1}, \mathrm{~L}_{7} \times \mathrm{T}_{2}$, $\mathrm{L}_{10} \times \mathrm{T}_{3}, \mathrm{~L}_{1} \times \mathrm{T}_{3}$ and $\mathrm{L}_{9} \times \mathrm{T}_{1}$. Hybrid $\mathrm{L}_{2} \times \mathrm{T}_{1}$ also exhibited higher magnitude of economic heterosis with higher mean performance. These hybrids $\mathrm{L}_{2} \times \mathrm{T}_{1}, \mathrm{~L}_{7} \times \mathrm{T}_{2}, \mathrm{~L}_{10} \times \mathrm{T} 3, \mathrm{~L}_{1} \times$ $T_{3}$, and $L_{9} x T_{1}$ were crosses between poor $x$ 
good gca effects of parent for grain yield per plant. Similar findings for identification of superior inbred lines, testers and hybrid based on gca and sca effects for grain yield and its component traits in maize were reported by Ismaeili et al., (2005), Choukan (2006), Luders et al., (2007), Lata et al., (2008), Alam et al., (2009), Jebaraj et al., (2010), Kanagarasu et al., (2010), Yousif and Sedeeq (2011), Guimaraes et al., (2012), Patil et al., (2012), Mural and Chikkalingaiah (2012), Abrha et al., (2013), Izhar and Chakraborty (2013), Panwar et al., (2013), Singh et al., (2013), Ulaganathan and Ibrahim (2014), Jahan et al., (2014), Rastgari et al., (2014) and Kumar et al., (2015). Luders et al., (2007), Dar et al., (2007), Kumari et al., (2008), Lata et al., (2008), Singh and Kumar (2009), Jebaraj et al., (2010), Reddy et al., (2011) and Motamedi et al., (2014) were reported for brown husk related traits and plant type traits in maize. Kooner et al., (2005), Singhal et al., (2006), Vijayabharathi et al., (2009), Kanagarasu et al., (2010), Lal et al., (2011), Rajitha et al., (2014) and Khan et al., (2016) were reported for grain yield and quality traits. which have shown high sca effects for grain yield involving parents of positive and significant gca effects can be exploited for the development of single cross hybrids since non additive gene action for most of the traits was observed. Further they can also be used for population improvement programme through reciprocal recurrent selection.

\section{References}

Abera, W., Rensburg, J.B. J-Van., Labuschagne, M. T. and Maartnes, H., 2004. Genotype environment interactions and yield stability of new maize in Ethiopia. South African J. Pl. Soil, 21(4): 251-254.

Abrha, S.W., Zeleke, H.Z. and Gissa, D.W. 2013. Line $x$ tester analysis of maize inbred lines for grain yield and yield related traits. Asian Journal of Plant Science and Research, 3(5): 12-19.

Abuali, A.I., Abdelmulla, A.A., Khalafalla, M.M., Idris, A.E. and Osman, A.M. 2012. Combining ability and heterosis for yield and yield components in maize (Zea mays L.). Australian Journal of Basic and Applied Sciences, 6(10): 36-41.

Alam, M.S. and Alam, M.F. 2009. Study on combining ability in inbred lines of maize using line $\mathrm{x}$ tester method. International Journal of Sustainable Agricultural Technology, 5(3): 32-36.

Anusheela,V., Muthiah, A.R. and Johnjoel, A. 2013. Heterosis in quality protein maize. International Journal of Scientific Research, 2(11): 567-570

Davenport, C.B. 1908. Degeneration, albinism and inbreeding. Science, 28: 454-455.

Davis, R.L. 1927. Report of plant breeder. Rep. Puarto Rico. Agric. Expt. Sta., 14-15.

Dubey, R.B., Joshi, V.N. and Pandiya, N.K. 2001. Heterosis and combining ability for quality, yield and maturity traits in conventional and non-conventional hybrids of maize (Zea mays L.). Indian J. Genet., 61(4): 353-355.

Dubey, R.B., Joshi, V.N., and Verma, M. 2009. Heterosis for nutritional quality and yield in conventional and nonconventional hybrids of maize (Zea mays L.). Indian J. Genet.,69(2): 109114.

East, E.M. 1908. Inbreeding in corn. Rept. Corn. Agric. Expt. Stat. for 1907. pp. 419-428.

Griffing, B. 1956. Concept of general and specific combining ability in relation to diallel crossing systems. Australian Journal of Biological Sciences, 9: 463493.

Griffing, B. 1956. Concept of general and 
specific combining ability in relation to diallel crossing systems. Australian Journal of Biological Sciences, 9: 463493.

Joshi, V.N., Sharma, G.S. and Ranwah, B.R. 1985. Prospects of Maize (Zea mays L.) as a new oil seed crop. In oil seed production, constraints and opportunities (Edited by H.C. Shrivastava and S. Bhaskaran). IBH Publi. Co., 529-533.

Kambe, G. R., Kage, U. K., Lohithaswa, H. C., Shekara, B.G. and Shobha, D. (2013). Combining ability studies in maize (Zea mays L.). Molecular Plant Breeding, 13: 116-127.

Kempthorne, O. 1957. An introduction to genetical statistics. John Willey and Sons Inc, New York, pp. 323-331.

Kempthorne, O. and Curnow, R.N. 1961. Partial diallel cross. Biometrics, 17: 229- 250.

Khan, S., Dadheech, A., Dubey, R. B. and Bharti, B. 2016. Combining ability and gene action studies for grain yield and quality parameters in yellow seeded maize (Zea mays L.) using line $\mathrm{x}$ tester crosses. International Journal of Bio-resource and Stress Management, 7(4) Special: 508-514.

Lal, M., Singh, D. and Dass, S. 2007. Studies for yield and quality traits in rabi in quality protein maize (Zea mays L.) Agric. Sci. Digest, 33: 245-247.

Lal, M., Singh, D. and Sain, D. 2011. General and specific combining ability

Lata, S., Guleria, S., Dev, J., Katna, G., Sood, B.C., Kalia, V. and Singh, A. 2010. Stability analysis in maize (Zea mays L.) hybrids across locations. Electronic Journal of Plant Breeding, 1 (3): 239- 243.

Manaik, P., Pavan, R., Nagabhusana, Hemareddy, H.B. and Lohithaswa, H.C. 2016.Genotype x environment interaction for yield and its attributing traits in single cross hybrids of maize (Zea mays L.). International Journal of Agriculture Sciences., 8:3231-323

Motamedi, M., Choukan, R., Hervan, E.M., Bihamta, M.R. and Kajouri, F.D. 2014. Investigation of genetic control for yield and related traits in maize (Zea mays L.) lines derived from temperate and subtropical germplasm. International Journal of Biosciences, 5(12): 123-129.

Premlatha, M., Kalamani, A. and Nirmalakumari, A. 2011. Heterosis and combining ability for grain yield and quality in maize (Zea mays L.). Advances in Environmental Biology, 5(6): 1264-1266.

Rawlings, J.O. and Cocherham, C.C. 1962. Triallel analysis, Crop Sci., 2: 228231.

Ruswandi, D., Supriatna, J., Makkulawu, A.T., Waluyo, B., Marta, H., Suryadi, E. and Ruswandi, S. 2015. Determination of combining ability and heterosis of grain yield components for maize mutants based on line $\mathrm{x}$ tester analysis. Asian Journal of Crop Science, 7: 19-33.

Sharief, A.E., El-Kalla, S.E., Gado, H.E. and Abo-Yousef, H.A.E. 2009. Heterosis in yellow maize. Australian Journal of Crop Science, 3(3): 146-154.

Singh, A., Shahi, J.P. and Langade, D.M. 2013. Combining ability studies for yield and its related traits in inbred lines of maize (Zea mays L.). Molecular Plant Breeding, 4(22): 177188.

Sumalini, K. 2012. Combining ability and heterosis for yield and quantitative traits in maize (Zea mays L.). Madras Agric. J., 99(4/6): 188-191.

Sumalini, K. and Shobha Rani, T. 2010. Heterosis and combining ability for polygenic traits in late maturing hybrids of maize (Zea mays L.). 
Madras Agric. J., 97: 340-343.

Tysdal, H.K., Kiesselbach, T.A. and Wtover,

H.L. 1942. Alfalfa breeding. Nebr. Agric. Expt. Sta. Res. Bull.124.

Vijayabharathi, A., Anandakumar, C.R. and

Gnanamalar, R.P. 2009. Combining ability analysis for yield and its components in popcorn (Zea mays var. everta Sturt.). Electronic Journal of Plant Breeding, 1(1): 28-32.

Yousif, A.M. and Sedeeq, F.A.Q. 2011. Estimation of combining ability for plant and ear height in maize. Tikrit Journal of Pure Science, 16(4). 31-34.

\section{How to cite this article:}

Deshraj Gurjar and Rambabu Dubey. 2020. Analysis of Heterosis and Combining Ability for Yield and Yield Contributing traits in Medium Maturity Hybrids of Maize (Zea mays L.). Int.J.Curr.Microbiol.App.Sci. 9(12): 1435-1459. doi: https://doi.org/10.20546/ijcmas.2020.912.172 